\title{
Low temperatures or high isolation delay increases the average COVID-19 infections in India : A Mathematical modeling approach
}

https://doi.org/10.1515/cmb-2020-0122

Received July 30, 2021; accepted August 23, 2021

\begin{abstract}
The dynamics of COVID-19 in India are captured using a set of delay differential equations by dividing a population into five compartments. The Positivity and Boundedness of the system is shown. The Existence and Uniqueness condition for the solution of system of equations is presented. The equilibrium points are calculated and stability analysis is performed. Sensitivity analysis is performed on the parameters of the model. Bifurcation analysis is performed and the critical delay is calculated. By formulating the spread parameter as a function of temperature, the impact of temperature on the population is studied. We concluded that with the decrease in temperature, the average infections in the population increases. In view of the coming winter season in India, there will be an increase in new infections. This model falls in line with the characteristics that increase in isolation delay increases average infections in the population.
\end{abstract}

Keywords: COVID-19; Isolation delay; Critical delay; Temperature; Average infections

MSC: 37N25; 92B05; $92 \mathrm{C} 99$

\section{Introduction}

The pandemic of COVID-19 has spread its tentacles across the world. Large number of countries are seeing a spike in the new infections and a few experiencing second wave of the pandemic. Moving into the winter season where influenza spreads rapidly and vaccine not being available to everyone in the near future, the impact of temperature and isolation is an important study in fighting COVID-19.

Various models were framed to understand the dynamics in population scale in India $[1,2,3,4]$. The impact of social distancing in optimal control of COVID-19 Epidemic spreading is studied in various models $[5,6,7,8]$. Various modeling frameworks captured the dynamics of diseases like dengue, Zika and COVID-19 $[9,10,11,12,13]$. Since COVID-19 is asymptomatic in many cases, immediate isolation of new infections is not practical. The isolated population includes home quarantined and hospitalized individuals. The delay involved in identification and effective isolation plays a major role in flattening the virus curve. A few models incorporated the impact delay on COVID-19 in India. Since the SARS-CoV-2 virus, responsible for COVID-19, spreads faster through air, the temperature in environment might play a role in spreading the virus. The effect of temperature on COVID-19 is studied in a few papers [14, 15, 16, 17, 18, 19, 20].

\footnotetext{
D Bhanu Prakash, Bishal Chhetri, Balasubramanian S: Department of Mathematics and Computer Science, Sri Sathya Sai Institute of Higher Learning - SSSIHL, India

*Corresponding Author: D K K Vamsi: Department of Mathematics and Computer Science, Sri Sathya Sai Institute of Higher Learning - SSSIHL, India, E-mail: dkkvamsi@sssihl.edu.in

Carani B Sanjeevi: Vice-Chancellor, Sri Sathya Sai Institute of Higher Learning - SSSIHL, India

Department of Medicine, Karolinska Institute, Stockholm, Sweden

Vice-Chancellor, Sri Sathya Sai Institute of Higher Learning - SSSIHL, India

E-mail: sanjeevi.carani@sssihl.edu.in, sanjeevi.carani@ki.se
} 
In this paper, we tried to capture the dynamics of COVID-19 in India using a system of delay differential equations from the inspiration given in [21]. The effect of temperature is also incorporated using the spread parameters. The rest of the paper goes in this fashion. In Section 2, we proposed the model along with the detailed explanations of delay, temperature and other parameters. In Section 3, a few important indicators like reproduction number and equilibrium points has been calculated. The stability of equilibrium points is discussed along with the Hopf bifurcation analysis in Section 4. In Section 5, the sensitivity analysis of sensitive parameters is given. The sensitivity analysis for the rest of the parameters is given in Appendix 1. Section 6 deals with numerical simulations. Section 7 presents the impact of temperature and isolation on COVID-19 in India. In Section 8, conclusions have been given.

\section{Mathematical Model}

We frame the model equations by considering the fraction of the people in each category. We assume a constant population $(\mathrm{N})$ and divide it into five compartments as:

- S - fraction of the total population that is healthy and has never caught the infection.

- E - fraction of the total population that is exposed to infection.

- I - fraction of the total population infected by the virus and undetected.

- Q - fraction of the total population that are found positive in the test and either hospitalized or quarantined.

- $\mathrm{R}$ - fraction of the total population that has recovered from the infection.

The proposed model is:

$$
\begin{aligned}
\frac{\mathrm{d} S(t)}{\mathrm{d} t} & =\Omega-\beta(T) S(t) I(t)-\mu S(t) \\
\frac{\mathrm{d} E(t)}{\mathrm{d} t} & =\beta(T) S(t) I(t)-\epsilon E(t)-\mu E(t) \\
\frac{\mathrm{d} I(t)}{\mathrm{d} t} & =\epsilon E(t)-\gamma I(t)-p e^{-\gamma \tau} I(t-\tau)-\mu I(t) \\
\frac{\mathrm{d} Q(t)}{\mathrm{d} t} & =p e^{-\gamma \tau} I(t-\tau)-\rho(1-\alpha) Q(t-\kappa)-\delta \alpha Q(t)-\mu Q(t) \\
\frac{\mathrm{d} R(t)}{\mathrm{d} t} & =\gamma I(t)+\rho(1-\alpha) Q(t-\kappa)-\mu R(t)
\end{aligned}
$$

Infectious hosts infect their neighbors at rate $\beta . \beta$ is the expected amount of people an infected person infects per day. That infected person can infect only $S(t)$ people. Hence the change of $S(t)$ to the next day $=-\beta$ $\mathrm{S}(\mathrm{t}) \mathrm{I}(\mathrm{t})$. Exposed individuals become infected at the rate $\epsilon$. If an infected individual remains infectious for $\tau$ units of time without having recovered, it enters a new state, $Q$ (for quarantine), with the detection probability p. Among I $(\mathrm{t}-\tau)$ infected persons at time $\mathrm{t}-\tau$, only a fraction of them $\left(e^{-\gamma \tau}\right)$ remains infectious and they are moved to quarantine $\mathrm{Q}$ at the rate $\mathrm{p}$. After staying in quarantine for $\kappa$ days, individuals in $\mathrm{Q}$ compartment move to R at the rate $\rho(1-\alpha)$, where $\rho$ is the rate at which people recover and $\alpha$ is the death rate. Individuals in Q compartment die at the rate $\delta \alpha$, where $\delta$ is the rate at which people die. Here, $\Omega$ and $\mu$ are the natural birth rate and natural death rate respectively.

All the parameters for this work are chosen in the Indian scenario from [2, 3, 4] making the results applicable in Indian context.

We incorporated two delays in our model [3, 22]. They are:

1. Time elapsed between infection and identification $(\tau)$ : Isolated individuals at time t were infected at time $\mathrm{t}-\tau$. This delay can be accounted for both delay in testing the asymptomatic individuals and the delay in isolating infected individuals.

2. Delay associated with recovery $(\kappa)$ : Quarantined individuals stay in quarantine for $\kappa$ days after which they are recovered. We consider it to be 14 days.

Among all the parameters in the proposed model, the transmission probability $\beta$ may depend on temperature $[2,18,19,23]$. We consider $\beta$ as a linear or quadratic function of temperature in similar lines with $[2,19]$ as 
Table 1: Table describing the parameter values

\begin{tabular}{|c|c|c|c|}
\hline Variable & Description & Value & Source \\
\hline$\Omega$ & Natural Birth rate & 0.062 & {$[4]$} \\
\hline$\epsilon$ & Rate of infection & 0.1961 & {$[2]$} \\
\hline$\frac{1}{\gamma}$ & Infectious period & 7 days & {$[2]$} \\
\hline$\frac{1}{\rho}$ & Quarantine Period & 14 days & {$[2]$} \\
\hline$\delta$ & Death Rate & 1 & {$[3]$} \\
\hline$\alpha$ & Death probability & $0.43 \%$ & {$[2]$} \\
\hline$\mu$ & Natural Death rate & 0.062 & {$[4]$} \\
\hline
\end{tabular}

follows :

$$
\begin{gathered}
\beta(T)=\beta_{0}+\beta_{1} T, \beta_{0}=0.84, \beta_{1}=-0.00425 \\
\left.\beta(T)=\beta_{0}-\beta_{1}\left(T-T_{M}\right)^{2}\right), \beta_{0}=0.792, \beta_{1}=0.000345, T_{M}=7.73
\end{gathered}
$$

Very few infected individuals at time 0 spreads the virus. So, we take initial value as $(\mathrm{S}(\mathrm{t}), \mathrm{E}(\mathrm{t}), \mathrm{I}(\mathrm{t}), \mathrm{Q}(\mathrm{t}), \mathrm{R}(\mathrm{t}), \mathrm{D}(\mathrm{t}))=(0.999,0,0.001,0,0,0)$.

The detection probability $(\sigma)$ ranges from 0 to 1 depending on the effectiveness of the system. For the preliminary analysis, we shall consider $\tau$ as 4 days [22] and p as 0.4 in the further sections.

\section{Mathematical Analysis}

\subsection{Positivity and Boundedness of solution:}

\subsubsection{Positivity of Solutions:}

For any mathematical model it is fundamental to show that the system of equations considered are positive and has bounded solutions. We now show that if the initial conditions of the system (1) - (5) are positive, then the solution remain positive for any future time.

Using the equations (1) - (5), we get,

$$
\begin{array}{ccc}
\left.\frac{\mathrm{d} S}{\mathrm{~d} t}\right|_{S=0}=\Omega \geq 0 & \left.\frac{\mathrm{d} E}{\mathrm{~d} t}\right|_{E=0}=\beta S I \geq 0 & \left.\frac{\mathrm{d} I}{\mathrm{~d} t}\right|_{I=0}=\epsilon E \geq 0 \\
\left.\frac{\mathrm{d} Q}{\mathrm{~d} t}\right|_{Q=0}=p e^{-\gamma \tau} I(t-\tau) \geq 0 & \left.\frac{\mathrm{d} R}{\mathrm{~d} t}\right|_{R=0}=\gamma I(t)+\rho(1-\alpha) Q(t-\kappa) \geq 0
\end{array}
$$

Thus all the above rates are non-negative on the bounding planes (given by $S=0, E=0, I=0, Q=0$, $\mathrm{R}=0$ ) of the non-negative region of the real space. So, if a solution begins in the interior of this region, it will remain inside it throughout time t. This happens because the direction of the vector field is always in the inward direction on the bounding planes as indicated by the above inequalities. Hence, we conclude that all the solutions of the the system (1) - (5) remain positive for any time $t>0$ provided that the initial conditions are positive. Next we will show that the solution is bounded with each of the bounded control variables. 


\subsubsection{Boundedness of Solutions:}

Let $\mathrm{N}(\mathrm{t})=\mathrm{S}(\mathrm{t})+\mathrm{E}(\mathrm{t})+\mathrm{I}(\mathrm{t})+\mathrm{Q}(\mathrm{t})+\mathrm{R}(\mathrm{t})$. Now,

$$
\begin{aligned}
\frac{\mathrm{d} N}{\mathrm{~d} t} & =\frac{\mathrm{d} S}{\mathrm{~d} t}+\frac{\mathrm{d} E}{\mathrm{~d} t}+\frac{\mathrm{d} I}{\mathrm{~d} t}+\frac{\mathrm{d} Q}{\mathrm{~d} t}+\frac{\mathrm{d} R}{\mathrm{~d} t} \\
& =\Omega-\delta \alpha Q(t)-\mu N(t) \leq \Omega-\mu N(t)
\end{aligned}
$$

Here the integrating factor is $e^{\mu t}$. Therefore after integration we get, $\mathrm{N}(\mathrm{t}) \leq \frac{\Omega}{\mu}+c e^{-\mu t}$. Now as $\mathrm{t} \rightarrow \infty$ we get, $\mathrm{N}(\mathrm{t}) \leq \frac{\Omega}{\mu}$.

Thus, we have shown that the system (1) - (5) is positive and bounded. Therefore the biologically feasible region is given by the following set,

$$
\Sigma=\left\{(S(t), E(t), I(t), Q(t), R(t)): N(t) \leq \frac{\Omega}{\mu}, t \geq 0\right\}
$$

\subsection{Existence and Uniqueness of Solution:}

For the general first order ODE of the form

$$
\dot{x}=f(t, x), \quad x\left(t_{0}\right)=x_{0}
$$

\subsubsection{Uniqueness of Solution:}

Let $\mathrm{D}$ denote the domain:

$$
\left|t-t_{0}\right| \leq a,|| x-x_{0}|| \leq b, x=\left(x_{1}, x_{2}, \ldots, x_{n}\right), x_{0}=\left(x_{10}, \ldots, x_{n 0}\right)
$$

and suppose that $f(t, x)$ satisfies the Lipschitz condition:

$$
\left\|f\left(t, x_{2}\right)-f\left(t, x_{1}\right)\right\| \leq k\left\|x_{2}-x_{1}\right\|
$$

and whenever the pairs $\left(t, x_{1}\right)$ and $\left(t, x_{2}\right)$ belong to the domain $D$, where $k$ is used to represent a positive constant.

Then, there exist a constant $\delta>0$ such that there exists a unique (exactly one) continuous vector solution $x(t)$ of the system (1) - (5) in the interval $\left|t-t_{0}\right| \leq \delta$. It is important to note that condition (8) is satisfied by requirement that:

$$
\frac{\partial f_{i}}{\partial x_{j}}, i, j=1,2, . ., n
$$

be continuous and bounded in the domain D.

\subsubsection{Existence of Solution:}

Let $D$ be the domain defined in 7 such that 8 hold. Then there exist a solution of model system of equations (1) - (5) which is bounded in the domain $D$.

Let:

$$
\begin{array}{cc}
f_{1} & =\Omega-\beta S I-\mu S \\
f_{2} & =\beta S I-(\epsilon+\mu) E \\
f_{3} & =\epsilon E-\left(\gamma+p e^{-\gamma \tau}+\mu\right) I \\
f_{4} & =p e^{-\gamma \tau} I-(\rho(1-\alpha)+\delta \alpha+\mu) Q \\
f_{5} & =\gamma I+\rho(1-\alpha) Q-\mu R
\end{array}
$$


We show that:

$$
\frac{\partial f_{i}}{\partial x_{j}}, i, j=1,2, \ldots, n
$$

is continuous and bounded in the domain $\mathrm{D}$. That is, the partial derivatives are continuous and bounded.

From equation (9) we have

$$
\begin{aligned}
& \frac{\partial f_{1}}{\partial S}=-\beta I-\mu,\left|\frac{\partial f_{1}}{\partial S}\right|=|-\beta I-\mu|<\infty \\
& \frac{\partial f_{1}}{\partial E}=0,\left|\frac{\partial f_{1}}{\partial E}\right|<\infty \\
& \frac{\partial f_{1}}{\partial I}=-\beta S,\left|\frac{\partial f_{1}}{\partial I}\right|=|-\beta S|<\infty \\
& \frac{\partial f_{1}}{\partial Q}=0,\left|\frac{\partial f_{1}}{\partial Q}\right|<\infty \\
& \frac{\partial f_{1}}{\partial R}=0,\left|\frac{\partial f_{1}}{\partial R}\right|<\infty
\end{aligned}
$$

Similarly, from equation (10) we also have that

$$
\begin{aligned}
& \frac{\partial f_{2}}{\partial S}=\beta I,\left|\frac{\partial f_{2}}{\partial S}\right|=|\beta I|<\infty \\
& \frac{\partial f_{2}}{\partial E}=-\epsilon-\mu,\left|\frac{\partial f_{2}}{\partial E}\right|=|-\epsilon-\mu|<\infty \\
& \frac{\partial f_{2}}{\partial I}=\beta S,\left|\frac{\partial f_{2}}{\partial I}\right|=|\beta S|<\infty \\
& \frac{\partial f_{2}}{\partial Q}=0,\left|\frac{\partial f_{2}}{\partial Q}\right|<\infty \\
& \frac{\partial f_{2}}{\partial R}=0,\left|\frac{\partial f_{2}}{\partial R}\right|<\infty
\end{aligned}
$$

Also from equation (11):

$$
\begin{aligned}
& \frac{\partial f_{3}}{\partial S}=0,\left|\frac{\partial f_{3}}{\partial S}\right|<\infty \\
& \frac{\partial f_{3}}{\partial E}=\epsilon,\left|\frac{\partial f_{3}}{\partial E}\right|=|\epsilon|<\infty \\
& \frac{\partial f_{3}}{\partial I}=-\left(\gamma+p e^{-\gamma \tau}+\mu\right),\left|\frac{\partial f_{3}}{\partial I}\right|=\left|-\left(\gamma+p e^{-\gamma \tau}+\mu\right)\right|<\infty \\
& \frac{\partial f_{3}}{\partial Q}=0,\left|\frac{\partial f_{3}}{\partial Q}\right|<\infty \\
& \frac{\partial f_{3}}{\partial R}=0,\left|\frac{\partial f_{3}}{\partial R}\right|<\infty
\end{aligned}
$$

Also from equation (12):

$$
\begin{aligned}
& \frac{\partial f_{4}}{\partial S}=0,\left|\frac{\partial f_{4}}{\partial S}\right|<\infty \\
& \frac{\partial f_{4}}{\partial E}=0,\left|\frac{\partial f_{4}}{\partial E}\right| \infty \\
& \frac{\partial f_{4}}{\partial I}=p e^{-\gamma \tau},\left|\frac{\partial f_{4}}{\partial I}\right|=\left|p e^{-\gamma \tau}\right|<\infty \\
& \frac{\partial f_{4}}{\partial Q}=-(\rho(1-\alpha)+\delta \alpha+\mu),\left|\frac{\partial f_{4}}{\partial Q}\right|=|-(\rho(1-\alpha)+\delta \alpha+\mu)|<\infty \\
& \frac{\partial f_{4}}{\partial R}=0,\left|\frac{\partial f_{4}}{\partial R}\right|<\infty
\end{aligned}
$$


Finally we have from equation (13) we have:

$$
\begin{aligned}
& \frac{\partial f_{5}}{\partial S}=0,\left|\frac{\partial f_{5}}{\partial S}\right|<\infty \\
& \frac{\partial f_{5}}{\partial E}=0,\left|\frac{\partial f_{5}}{\partial E}\right|<\infty \\
& \frac{\partial f_{5}}{\partial I}=\gamma,\left|\frac{\partial f_{5}}{\partial I}\right|=|\gamma|<\infty \\
& \frac{\partial f_{5}}{\partial Q}=\rho(1-\alpha),\left|\frac{\partial f_{5}}{\partial Q}\right|=|\rho(1-\alpha)|<\infty \\
& \frac{\partial f_{5}}{\partial R}=-\mu,\left|\frac{\partial f_{5}}{\partial R}\right|=|-\mu|<\infty
\end{aligned}
$$

Hence we have shown that all the partial derivatives are continuous and bounded. Therefore, Lipschitz condition is satisfied. Hence, by above paragraph on uniqueness of solutions, there exists a unique solution of system (1) - (5) in the region $D$.

\subsection{Reproduction Number $\left(\mathcal{R}_{0}\right)$ :}

The basic reproductive number is the mean number of secondary cases that a typical infected case will cause in a population with no immunity to the disease in the absence of interventions to control the infection.

The variational matrix of the model computed at the infection free state $(S=1, E=I=Q=R=0)$ gives the non-negative new infection F-matrix and non-singular transition V-matrix respectively as follows :

$$
F=\left[\begin{array}{ll}
0 & \beta \\
0 & 0
\end{array}\right], V=\left[\begin{array}{cc}
\epsilon+\mu & 0 \\
-\epsilon & \mu+\gamma+p e^{-\gamma \tau}
\end{array}\right]
$$

The basic reproduction number $\mathcal{R}_{0}=\rho\left(F V^{-1}\right)$, where $\rho\left(F V^{-1}\right)$ is the spectral radius for a next generation matrix $F V^{-1}[1]$ is given as:

$$
\mathcal{R}_{0}=\frac{\beta \epsilon}{(\epsilon+\mu)\left(\gamma+p e^{-\gamma \tau}+\mu\right)}
$$

The effect of $\beta, p, \tau$ on $\mathcal{R}_{0}$ is discussed in the following Figures 1,2,3. These plots are simulated using dde23 method in MATLAB. 

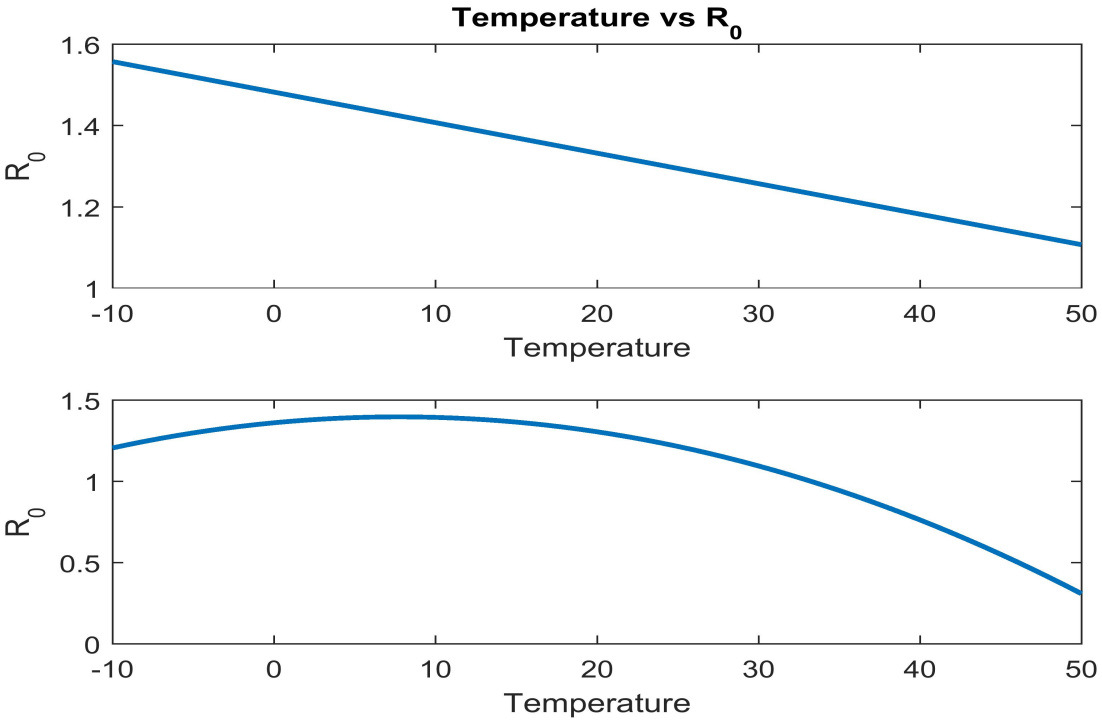

Figure 1: Impact of temperature $(\mathrm{T})$ on $\mathcal{R}_{0}$

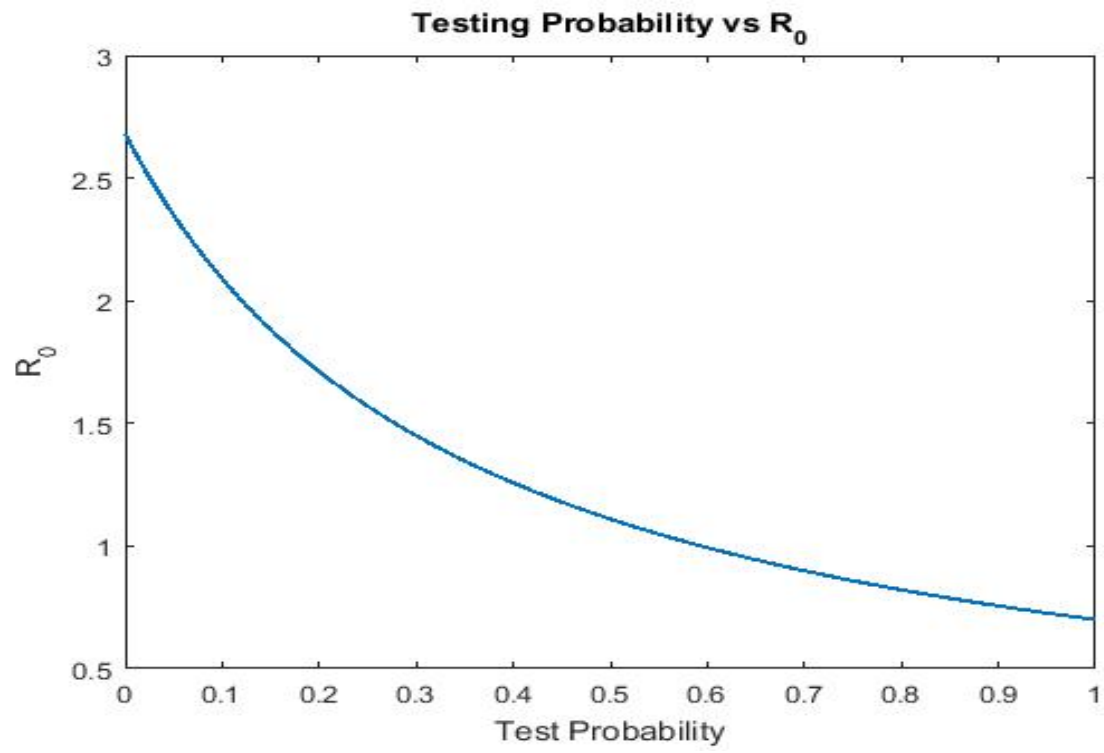

Figure 2: Impact of isolation probability $(\mathrm{p})$ on $\mathcal{R}_{0}$

\subsection{Equilibrium states:}

There are two equilibrium points of the delay model which are as follows.

The trivial equilibrium point, $\mathrm{E}_{0}=(1,0,0,0,0)$. 


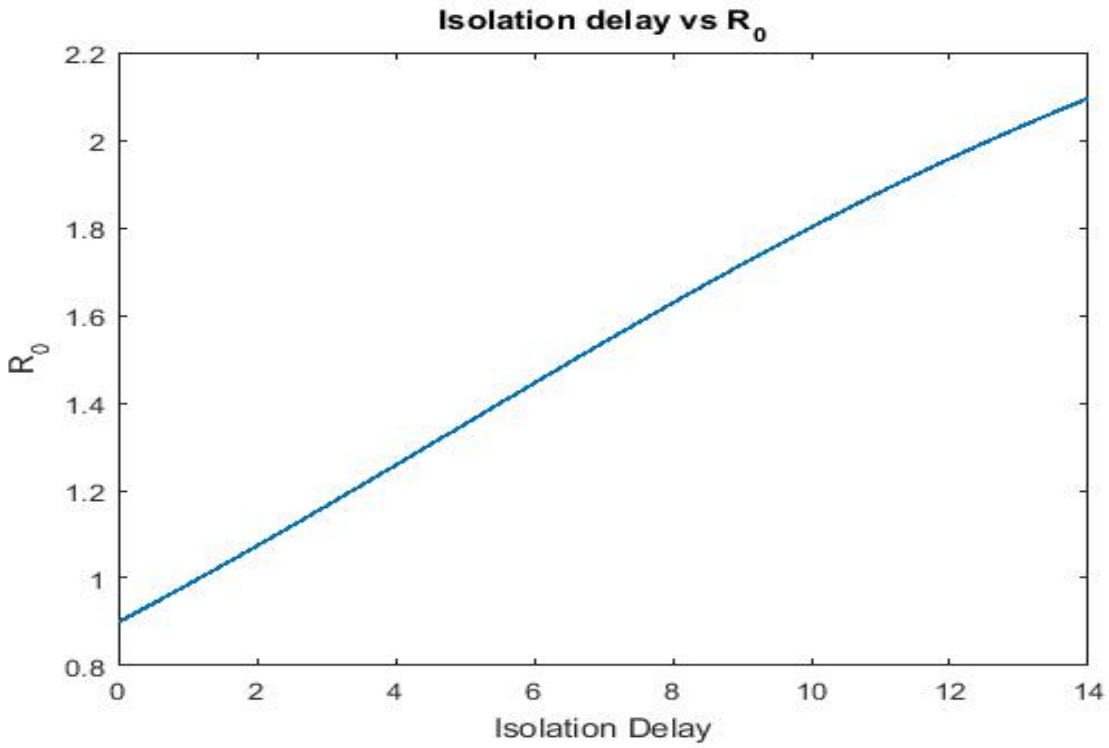

Figure 3: Impact of isolation $\operatorname{delay}(\tau)$ on $\mathcal{R}_{0}$

The endemic equilibrium is given as:

$$
\begin{aligned}
S^{\star} & =\frac{1}{\beta \epsilon}(\epsilon+\mu)\left(\gamma+p e^{-\gamma \tau}+\mu\right) \\
E^{\star} & =\frac{\mu-\mu S^{\star}}{\epsilon+\mu} \\
I^{\star} & =\frac{\epsilon}{\mu+\gamma+p e^{-\gamma \tau}} E^{\star} \\
Q^{\star} & =\frac{p e^{-\gamma \tau}}{\rho(1-\alpha)+\delta \alpha+\mu} I^{\star} \\
R^{\star} & =\frac{\gamma I^{\star}+\rho(1-\alpha) Q^{\star}}{\mu}
\end{aligned}
$$

\section{Condition for existence of $E^{\star}$}

This equilibrium exists when it satisfies all the initial conditions. So Endemic equilibrium exists if and only if $E^{\star}>0$. This implies that $S^{\star}<1$. Hence the condition is $(\epsilon+\mu)\left(\gamma+p e^{-\gamma \tau}+\mu\right)<\beta \epsilon$.

\section{Stability Analysis}

To perform the stability analysis, the Jacobian matrix[J] for the proposed system is computed as:

$$
J[E]=\left[\begin{array}{ccccc}
-\beta I-\mu & 0 & -\beta S & 0 & 0 \\
\beta I & -\mu-\epsilon & \beta S & 0 & 0 \\
0 & \epsilon & -\gamma-\mu-p e^{-\gamma \tau} e^{-\lambda \tau} & 0 & 0 \\
0 & 0 & p e^{-\gamma \tau} e^{-\lambda \tau} & -\rho(1-\alpha) e^{-\kappa \lambda}-\delta \alpha-\mu & 0 \\
0 & 0 & \gamma & -\rho(1-\alpha) e^{-\kappa \lambda} & -\mu
\end{array}\right]
$$

The characteristic equation is given as:

$$
\chi(\lambda)=(\lambda+\mu)\left(\lambda+\mu+\delta \alpha+\rho(1-\alpha) e^{-\kappa \lambda}\right)\left(\lambda^{3}+a_{1} \lambda^{2}+a_{2} \lambda+a_{3}+e^{-\lambda \tau}\left(b_{0} \lambda^{2}+b_{1} \lambda+b_{2}\right)\right)
$$


where,

$$
\begin{aligned}
& a_{1}=3 \mu+\epsilon+\gamma+\beta I \\
& a_{2}=(\gamma+\mu)(\epsilon+\mu)+(\gamma+\epsilon+2 \mu)(\beta I+\mu)-\epsilon \beta S \\
& a_{3}=(\gamma+\mu)(\epsilon+\mu)(\beta I+\mu)-\beta \epsilon \mu S \\
& b_{0}=p e^{-\gamma \tau} \\
& b_{1}=p e^{-\gamma \tau}(2 \mu+\epsilon+\beta I) \\
& b_{2}=p e^{-\gamma \tau}(\epsilon+\mu)(\mu+\beta I)
\end{aligned}
$$

\subsection{Stability of Infection-free $\operatorname{state}\left(\mathrm{E}_{0}\right)$ :}

The characteristic equation about the infection-free state $\left(\mathrm{E}_{0}\right)$ is given as:

$$
\chi(\lambda)=(\lambda+\mu)^{2}\left(\lambda+\mu+\delta \alpha+\rho(1-\alpha) e^{-\kappa \lambda}\right)\left(\lambda^{2}+d_{1} \lambda+d_{2}+e^{-\lambda \tau}\left(e_{1} \lambda+e_{2}\right)\right)
$$

where,

$$
\begin{aligned}
& d_{1}=2 \mu+\epsilon+\gamma \\
& d_{2}=(\gamma+\mu)(\epsilon+\mu)-\epsilon \beta \\
& e_{1}=p e^{-\gamma \tau} \\
& e_{2}=p e^{-\gamma \tau}(\mu+\epsilon)
\end{aligned}
$$

Now the characteristic equation without delays $(\tau=0, \kappa=0)$ is given as,

$$
\chi(\lambda)=(\lambda+\mu)^{2}(\lambda+\mu+\delta \alpha+\rho(1-\alpha))\left(\lambda^{2}+d_{1} \lambda+d_{2}+\left(e_{1} \lambda+e_{2}\right)\right)
$$

Using Descartes rule of sign change we see that all the roots of (15) are negative, which implies that the disease free equilibrium $E_{0}$ is locally asymptotically stable with $\alpha<1$ and $(\gamma+\mu)(\epsilon+\mu)>\epsilon \beta$.

Now we will analyze the stability of $E_{0}$ with delays. Let us first consider the following,

$$
(\lambda+\mu)^{2}\left(\lambda+\mu+\delta \alpha+\rho(1-\alpha) e^{-\kappa \lambda}\right)=0
$$

The two roots of this equation are $: \lambda_{1,2}=-\mu$, and others are the roots of the following equation.

$$
\left(\lambda+\mu+\delta \alpha+\rho(1-\alpha) e^{-\kappa \lambda}\right)=0
$$

Let $\lambda=i \omega$. Substituting in the above equation (17) and simplifying the terms and equating the real parts and imaginary parts, we get,

$$
\begin{aligned}
\omega & =\rho(1-\alpha \cos \kappa \omega) \\
\mu+\delta \alpha & =\rho(1-\alpha \sin \kappa \omega)
\end{aligned}
$$

Squaring and adding the above two equations give

$$
\omega^{2}+(\mu+\delta \alpha)^{2}-\rho^{2}(1-\alpha)^{2}=0
$$

If $(\mu+\delta \alpha)^{2}>\rho^{2}(1-\alpha)^{2}$ then all roots of (18) are imaginary, which implies that all roots of (17) are negative. So far now we have all the roots of (16) to be negative provided $(\mu+\delta \alpha)^{2}>\rho^{2}(1-\alpha)^{2}$.

Now we consider the following expression of (14) and state and prove the bifurcation theorem for $E_{0}$.

$$
\left(\lambda^{2}+d_{1} \lambda+d_{2}+e^{-\lambda \tau}\left(e_{1} \lambda+e_{2}\right)\right)=0
$$




\section{Theorem}

Let $(\mu+\delta \alpha)^{2}>(1-\alpha)^{2} \rho^{2}$ and $f_{1}>0$ and $E_{0}$ be asymptotically stable without any delay. Then

1. When the conditions $d_{2}^{2}<e_{2}^{2}, \gamma^{2}>\omega^{2}, d_{2}<\omega^{2}$ and $x>y$ holds, the disease free equilibrium point $E_{0}^{\star}$ of the delayed model (2.1) is locally asymptotically stable while $\tau<\tau^{\star}$ and is unstable while $\tau>\tau^{\star}$ where

$$
\tau^{\star}=\frac{1}{\omega_{0}} \arccos \left[\frac{\left(e_{2}-d_{1} e_{1}\right) \omega^{2}-d_{2} e_{2}}{e_{1}^{2} \omega^{2}+e_{2}^{2}}\right]
$$

For $\tau=\tau^{\star}$, Hopf Bifurcation occurs as the value of $\tau$ passes through the critical value $\tau^{\star}$.

2. If $d_{2}^{2}>e_{2}^{2}$, then $E_{0}$ is locally asymptotically stable for all $\tau$.

Proof: The disease free equilibrium point $E_{0}$ of the delayed system (2.1) will be asymptotically stable if all the roots of the characteristic equation (19) have negative real parts. We have seen that $E_{0}$ of the non delayed system is asymptotically stable. We now check the sign of the real part of the roots of (19) as we vary the value of the gestation delay $\tau$.

Let $\lambda=\mu(\tau)+i \omega(\tau)$ where, $\mu$ and $\omega$ are real. Since $E_{0}$ is stable for the non-delayed system and $\mu(0)<0$, we will choose $\tau$ sufficiently close to 0 and use continuity of $\tau$ to prove the theorem. Let $\tau>0$ be sufficiently small, then by continuity $\mu(\tau)<0$ and $E^{\star}$ will still remain stable. The stability changes for some values of $\tau$ for which $\mu(\tau)=0$ and $\omega(\tau) \neq 0$ that is when $\lambda$ is purely imaginary. Let $\tau^{\star}$ be such that $\mu\left(\tau^{*}\right)=0$ and $\omega\left(\tau^{\star}\right) \neq 0$. In this case the steady state loses stability and becomes unstable when $\mu(\tau)$ becomes positive.

By Rouche's theorem [24] the transcendental equation (14) has roots with positive real parts if and only if it has purely imaginary roots. Now we will assume that the characteristic equation (19) has purely imaginary roots and then arrive at a contradiction.

Let $\lambda=i \omega$. Substituting in the above equation, simplifying the terms and equating the real parts and imaginary parts, we get,

$$
\begin{aligned}
d_{2}-\omega^{2} & =\left(-e_{1} \omega\right) \sin (\omega \tau)-e_{2} \cos (\omega \tau) \\
d_{1} \omega & =\left(-e_{1} \omega\right) \cos (\omega \tau)+e_{2} \sin (\omega \tau)
\end{aligned}
$$

Squaring and adding the above two equations give

$$
\omega^{4}+f_{1} \omega^{2}+f_{2}=0
$$

where,

$$
\begin{aligned}
& f_{1}=d_{1}^{2}-2 d_{2}-e_{1}^{2} \\
& f_{2}=d_{2}^{2}-e_{2}^{2}
\end{aligned}
$$

If $d_{2}^{2}>e_{2}^{2}$ then all roots of (20) are imaginary. This contradicts that $\omega$ is real therefore equation (19) does not have purely imaginary roots. Hence by Rouche's Theorem we conclude that all roots of (19) have negative real parts. Therefore the disease free equilibrium point $E_{0}$ is asymptotically stable for all $\tau$, whenever $d_{2}^{2}>e_{2}^{2}$.

When $d_{2}^{2}<e_{2}^{2}$ from equation (20), we see that there is only one sign change, therefore using Descartes rule of sign change we conclude that whenever $d_{2}^{2}<e_{2}^{2}$ equation (20) has unique positive root say $\omega^{\star}$. This implies that the characteristics equation (19) has a purely imaginary roots $\pm i \omega^{\star}$. Now we will calculate the critical value of the delay $\tau^{\star}$ based on $\omega^{\star}$.

Calculation of $\tau^{*}$ at $\omega^{*}$ :

Consider,

$$
\begin{aligned}
d_{2}-\omega^{\star 2} & =\left(-e_{1} \omega^{\star}\right) \sin \left(\omega^{\star} \tau\right)-e_{2} \cos \left(\omega^{\star} \tau\right) \\
d_{1} \omega^{\star} & =\left(-e_{1} \omega^{\star}\right) \cos \left(\omega^{\star} \tau\right)+e_{2} \sin \left(\omega^{\star} \tau\right)
\end{aligned}
$$


Solving the above equation for $\tau^{\star}$ we get,

$$
\tau^{\star}=\frac{1}{\omega^{\star}} \cos ^{-1}\left[\frac{\left(e_{2}-d_{1} e_{1}\right) \omega^{2}-d_{2} e_{2}}{e_{1}^{2} \omega^{2}+e_{2}^{2}}\right]
$$

Now to show that there is at least one eigen value with a positive real part for $\tau>\tau^{\star}$, we verify the transversality condition $\frac{d(\operatorname{Re} \lambda(\tau))}{d \tau}>0$ at $\tau=\tau^{\star}$

From (19) we have,

$$
\lambda^{2}+d_{1} \lambda+d_{2}+e^{-\lambda \tau}\left(e_{1} \lambda+e_{2}\right)=0
$$

substituting for $e_{1}$ and $e_{2}$ we get

$$
\lambda^{2}+d_{1} \lambda+d_{2}+(\lambda \rho+\rho(\mu+\epsilon)) e^{-(\lambda+\gamma) \tau}=0
$$

Differentiating the above equation with respect to $\tau$, we get,

$$
\begin{gathered}
\left(2 \lambda+d_{1}+\rho e^{-(\lambda+\gamma) \tau}-\lambda \rho \tau(\lambda+\gamma) e^{-(\lambda+\gamma) \tau}-\rho \tau(\mu+\epsilon)(\lambda+\gamma) e^{-(\lambda+\gamma) \tau}\right) \frac{d \lambda}{d \tau} \\
=\lambda \rho(\lambda+\gamma)^{2} e^{-(\lambda+\gamma) \tau}+\rho(\mu+\epsilon)(\lambda+\gamma)^{2} e^{-(\lambda+\gamma)}
\end{gathered}
$$

simplifying we get,

$$
\left(\frac{d \lambda}{d \tau}\right)^{-1}=\frac{2 \lambda+d_{1}}{\rho(\lambda+\gamma)^{2}(\lambda+\mu+\epsilon) e^{-(\lambda+\gamma) \tau}}+\frac{1-\tau(\lambda+\gamma)(\lambda+\mu+\epsilon)}{(\lambda+\gamma)^{2}(\lambda+\mu+\epsilon)}
$$

From (22) we get,

$$
e^{-(\lambda+\gamma) \tau}=\frac{-\lambda^{2}-d_{1} \lambda-d_{2}}{\rho(\mu+\epsilon)+\lambda \rho}
$$

substituting the expression for $e^{-(\lambda+\gamma) \tau}$ in (23) we get,

$$
\left(\frac{d \lambda}{d \tau}\right)^{-1}=\frac{-\left(2 \lambda+d_{1}\right)}{(\lambda+\gamma)^{2}\left(\lambda^{2}+d_{1} \lambda+d_{2}\right)}+\frac{1}{(\lambda+\gamma)^{2}(\lambda+\mu+\epsilon)}-\frac{\tau}{(\lambda+\gamma)}
$$

At $\tau=\tau^{\star}$ and $\lambda=i \omega^{\star}$

$$
\left(\frac{d \lambda}{d \tau}\right)^{-1}=\frac{-\left(2 i \omega+d_{1}\right)}{(i \omega+\gamma)^{2}\left((i \omega)^{2}+d_{1} i \omega+d_{2}\right)}+\frac{1}{(i \omega+\gamma)^{2}(i \omega+\mu+\epsilon)}-\frac{\tau}{(i \omega+\gamma)}
$$

Now considering only the real parts and simplifying we get,

$$
\left(\operatorname{Re}\left(\frac{d \lambda}{d \tau}\right)^{-1}\right)=\frac{d_{1}\left(-\gamma^{2}+\omega^{2}\right)\left(d_{2}-\omega^{2}\right)+2 \omega^{2} d_{1} \gamma\left(2+d_{1}\right)}{\left(\left(\gamma^{2}-\omega^{2}\right)^{2}+4 \omega^{2} \gamma^{2}\right)\left(\left(d_{2}-\omega^{2}\right)^{2}+d_{1} \omega^{2}\right)}+\frac{\left(\gamma^{2}-\omega^{2}\right)(\mu+\epsilon)-2 \omega^{2} \gamma}{\left(\left(\gamma^{2}-\omega^{2}\right)^{2}+4 \omega^{2} \gamma^{2}\right)\left((\mu+\epsilon)^{2}+\omega^{2}\right)}-\frac{\tau^{\star} \gamma}{\left(\gamma^{2}+\omega^{2}\right)}
$$

Therefore, we have,

$$
\operatorname{sign}\left(\operatorname{Re}\left(\frac{d \lambda}{d \tau}\right)^{-1}\right)>0
$$

provided we have $(x+y)>0$ and $x+y>z$, where,

$$
\begin{gathered}
x=\frac{d_{1}\left(-\gamma^{2}+\omega^{2}\right)\left(d_{2}-\omega^{2}\right)+2 \omega^{2} d_{1} \gamma\left(2+d_{1}\right)}{\left(\left(\gamma^{2}-\omega^{2}\right)^{2}+4 \omega^{2} \gamma^{2}\right)\left(\left(d_{2}-\omega^{2}\right)^{2}+d_{1} \omega^{2}\right)} \\
y=\frac{\left(\gamma^{2}-\omega^{2}\right)(\mu+\epsilon)-2 \omega^{2} \gamma}{\left(\left(\gamma^{2}-\omega^{2}\right)^{2}+4 \omega^{2} \gamma^{2}\right)\left((\mu+\epsilon)^{2}+\omega^{2}\right)} \\
z=\frac{\tau^{\star} \gamma}{\left(\gamma^{2}+\omega^{2}\right)}
\end{gathered}
$$

Hence with the above conditions transversality Condition holds. Therefore this implies that there is at least one eigenvalue with positive real part for $\tau>\tau^{\star}$. Hence Hopf Bifurcation occurs at $\tau=\tau^{\star}$. 


\subsection{Stability of Endemic state( $\left.E^{\star}\right)$}

The characteristic equation about the infection-free state $\left(E^{\star}\right)$ is given as:

$$
\chi(\lambda)=(\lambda+\mu)\left(\lambda+\mu+\delta \alpha+\rho(1-\alpha) e^{-\kappa \lambda}\right)\left(\lambda^{3}+a_{1} \lambda^{2}+a_{2} \lambda+a_{3}+e^{-\lambda \tau}\left(b_{0} \lambda^{2}+b_{1} \lambda+b_{2}\right)\right)
$$

where,

$$
\begin{aligned}
& a_{1}=3 \mu+\epsilon+\gamma+\mu\left(\mathcal{R}_{0}-1\right) \\
& a_{2}=(\gamma+\epsilon+2 \mu) \mu \mathcal{R}_{0}-p e^{-\gamma \tau}(\epsilon+\mu) \\
& a_{3}=\mu(\epsilon+\mu)\left[(\gamma+\mu)\left(\mathcal{R}_{0}-1\right)-p e^{-\gamma \tau}\right] \\
& b_{0}=p e^{-\gamma \tau} \\
& b_{1}=p e^{-\gamma \tau}\left(\mu+\epsilon+\mu \mathcal{R}_{0}\right) \\
& b_{2}=p e^{-\gamma \tau}(\epsilon+\mu) \mu \mathcal{R}_{0}
\end{aligned}
$$

Now the characteristic equation without delays $(\tau=0, \kappa=0)$ is given as,

$$
\chi(\lambda)=(\lambda+\mu)(\lambda+\mu+\delta \alpha+\rho(1-\alpha))\left(\lambda^{3}+\left(a_{1}+b_{0}\right) \lambda^{2}+\left(a_{2}+b_{1}\right) \lambda+a_{3}+b_{2}\left(e_{1} \lambda+e_{2}\right)\right)
$$

Using Descartes rule of sign change we see that all the roots of (24) are negative provided we have,

$$
\begin{aligned}
& \text { 1. } \alpha<1 \\
& \text { 2. }\left(a_{1}+b_{0}\right)>0,\left(b_{1}+a_{2}\right)>0 \text {, and }\left(a_{3}+b_{2}\right)>0
\end{aligned}
$$

Therefore with the above conditions the infected equilibrium $E_{1}$ to be locally asymptotically stable without any delay.

Now we will analyze the stability of $E_{0}$ with delays. Let us first consider the following,

$$
(\lambda+\mu)\left(\lambda+\mu+\delta \alpha+\rho(1-\alpha) e^{-\kappa \lambda}\right)=0
$$

The two roots of this equation are : $\lambda_{1}=-\mu$, and others are the roots of the following equation.

$$
\left(\lambda+\mu+\delta \alpha+\rho(1-\alpha) e^{-\kappa \lambda}\right)=0
$$

Let $\lambda=i \omega$. Substituting in the above equation (26) and simplifying the terms and equating the real parts and imaginary parts, we get,

$$
\begin{aligned}
\omega & =\rho(1-\alpha \cos \kappa \omega) \\
\mu+\delta \alpha & =\rho(1-\alpha \sin \kappa \omega)
\end{aligned}
$$

Squaring and adding the above two equations give

$$
\omega^{2}+(\mu+\delta \alpha)^{2}-\rho^{2}(1-\alpha)^{2}=0
$$

If $(\mu+\delta \alpha)^{2}>\rho^{2}(1-\alpha)^{2}$ then all roots of (27) are imaginary, which implies that all roots of (26) are negative. So far now we have all the roots of (25) to be negative provided $(\mu+\delta \alpha)^{2}>\rho^{2}(1-\alpha)^{2}$.

Now consider the following,

$$
\left(\lambda^{3}+a_{1} \lambda^{2}+a_{2} \lambda+a_{3}+e^{-\lambda \tau}\left(b_{0} \lambda^{2}+b_{1} \lambda+b_{2}\right)\right)=0
$$

Let $\lambda=i \omega$. Substituting in the above equation gives:

$$
-i \omega^{3}-a_{1} \omega^{2}+a_{2} i \omega+a_{3}(\cos \omega \tau-i \sin \omega \tau)\left(-b_{0} \omega^{2}+b_{1} i \omega+b_{2}=0\right.
$$


Simplifying the terms and equating the real parts and imaginary parts, we get,

$$
\begin{aligned}
a_{3}-a_{1} \omega^{2} & =\left(-a_{3} b_{1} \omega\right) \sin (\omega \tau)+\left(a_{3} b_{0} \omega^{2}-a_{3} b_{2}\right) \cos (\omega \tau) \\
a_{2} \omega-\omega^{3} & =\left(-a_{3} b_{1} \omega\right) \cos (\omega \tau)-\left(a_{3} b_{0} \omega^{2}-a_{3} b_{2}\right) \sin (\omega \tau)
\end{aligned}
$$

Squaring and adding the above two equations give

$$
\omega^{6}+c_{1} \omega^{4}+c_{2} \omega^{2}+c_{3}=0
$$

where,

$$
\begin{aligned}
& c_{1}=a_{1}^{2}-2 a_{2}-a_{3}^{2} b_{0}^{2} \\
& c_{2}=a_{2}^{2}+2 a_{3}^{2} b_{0} b_{2}-a_{3}^{2} b_{1}^{2}-2 a_{1} a_{3} \\
& c_{3}=a_{3}^{2}\left(1-b_{2}^{2}\right)
\end{aligned}
$$

Now if $c_{1}>0, c_{2}>0, c_{3}>0$ then (29) has no real roots. This implies that the characteristic equation (28) has all roots to be negative. Therefore $E^{\star}$ is asymptotically stable. Where if $c_{3}<0$ then we have $E^{\star}$ to be unstable. Thus from the above analysis we conclude that $E^{\star}$ is asymptotically stable provided:

$$
\begin{aligned}
& \text { 1. }(\mu+\delta \alpha)^{2}>\rho^{2}(1-\alpha)^{2} \\
& \text { 2. } c_{1}>0, c_{2}>0, c_{3}>0
\end{aligned}
$$

If any of the above two condition is violated then $E^{\star}$ should change its nature of being stable. Figure 7 and Figure 8 depicts the dynamics of the system for two specific values of $\beta$. We will illustrate this by performing numerical simulations in section 6.

\section{Sensitivity Analysis}

In this section we check the sensitivity of all the parameters of the proposed model. As each parameter is varied in different intervals, the total infected cell population, mean infected cell population and the mean square error are plotted with respect to time. These plots are used to determine the sensitivity of the parameter. The different intervals chosen are given in the following Table 2. The fixed parameter values are taken from Table 1.

\subsection{Parameter $\boldsymbol{\beta}$}

The results related to sensitivity of $\beta$, varied in three intervals as mentioned in table 2, are given in Figure 4. The plots of infected population for each varied value of the parameter $\beta$ per interval, the mean infected population and the mean square error are used to determine the sensitivity. We conclude from these plots that the parameter $\beta$ is not sensitive in interval II and sensitive in I .

\subsection{Parameter $\boldsymbol{\omega}$}

The results related to sensitivity of $\omega$, varied in two intervals as mentioned in table 2, are given in Figure 5. The plots of infected population for each varied value of the parameter $\omega$ per interval, the mean infected population and the mean square error are used to determine the sensitivity. We conclude from these plots that the parameter $\omega$ is sensitive in both interval I and II. 
Table 2: Sensitivity Analysis

\begin{tabular}{|c|c|c|}
\hline Parameter & Interval & Step Size \\
\hline \multirow[t]{2}{*}{$\mu$} & 0 to 0.5 & \multirow[t]{2}{*}{0.01} \\
\hline & .5 to 2.5 & \\
\hline \multirow[t]{2}{*}{$\bar{\beta}$} & 0 to .5 & \multirow[t]{2}{*}{0.01} \\
\hline & 2 to 3 & \\
\hline \multirow[t]{2}{*}{$\alpha$} & 0 to 2 & \multirow[t]{2}{*}{0.01} \\
\hline & 2 to 5 & \\
\hline \multirow[t]{2}{*}{$\gamma$} & 0 to 1 & \multirow[t]{2}{*}{0.01} \\
\hline & 1 to 2.5 & \\
\hline \multirow[t]{2}{*}{$\epsilon$} & 0 to 0.5 & \multirow[t]{2}{*}{0.01} \\
\hline & 1.5 to 2.5 & \\
\hline \multirow[t]{2}{*}{$\omega$} & 0 to 2 & \multirow[t]{2}{*}{0.01} \\
\hline & 2 to 4 & \\
\hline \multirow[t]{2}{*}{$\delta$} & 0 to 1 & \multirow[t]{2}{*}{0.01} \\
\hline & 1 to 2.5 & \\
\hline
\end{tabular}
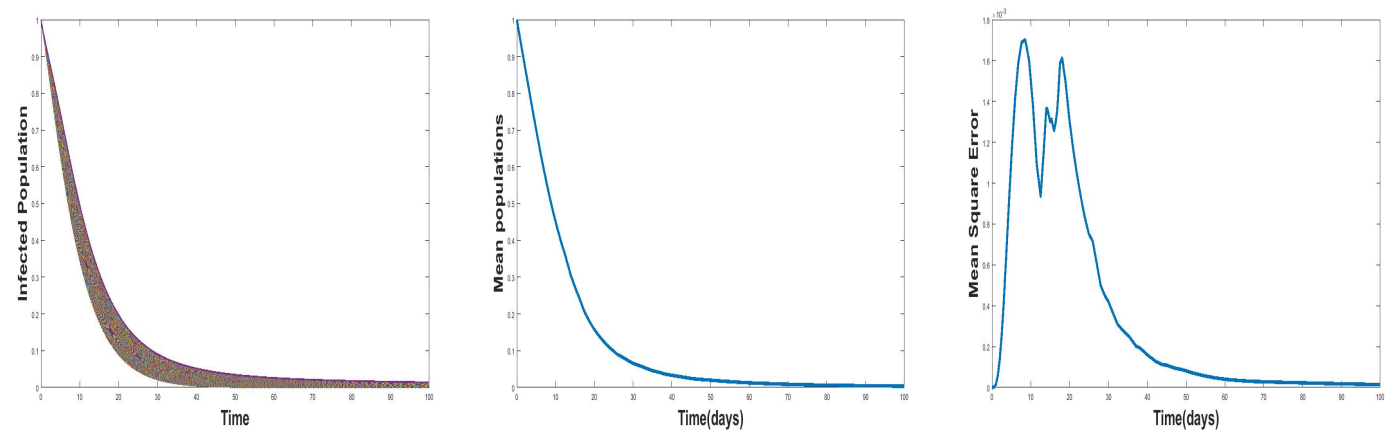

(a) Interval I: 0 to 0.5
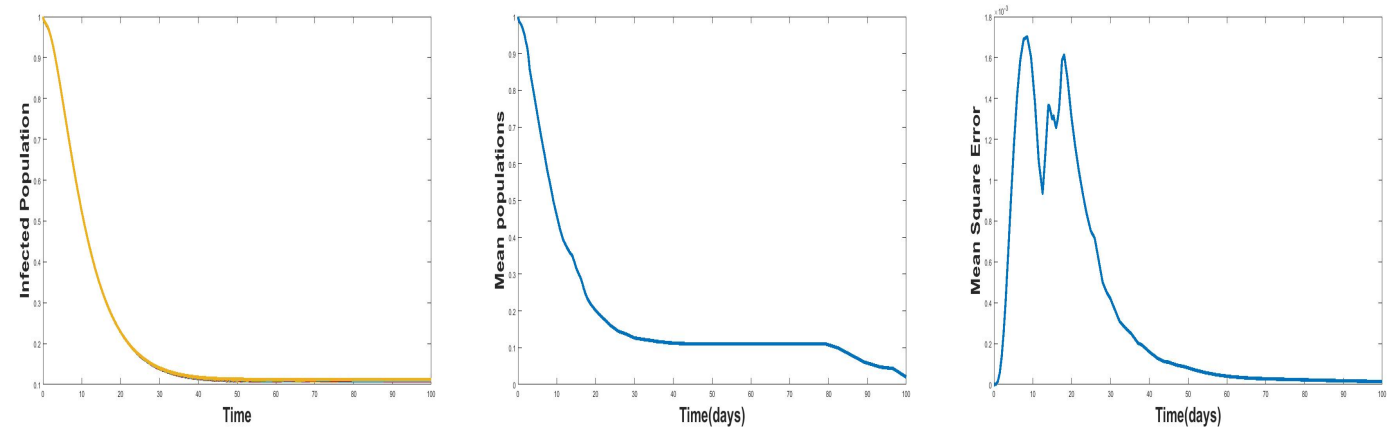

(b) Interval II : 2 to 3

Figure 4: Figure depicting the sensitivity Analysis of $b_{1}$ varied in two intervals in table 2. The plots depict the infected population for each varied value of the parameter $\beta$ per interval along with the mean infected population and the mean square error in the same interval. 

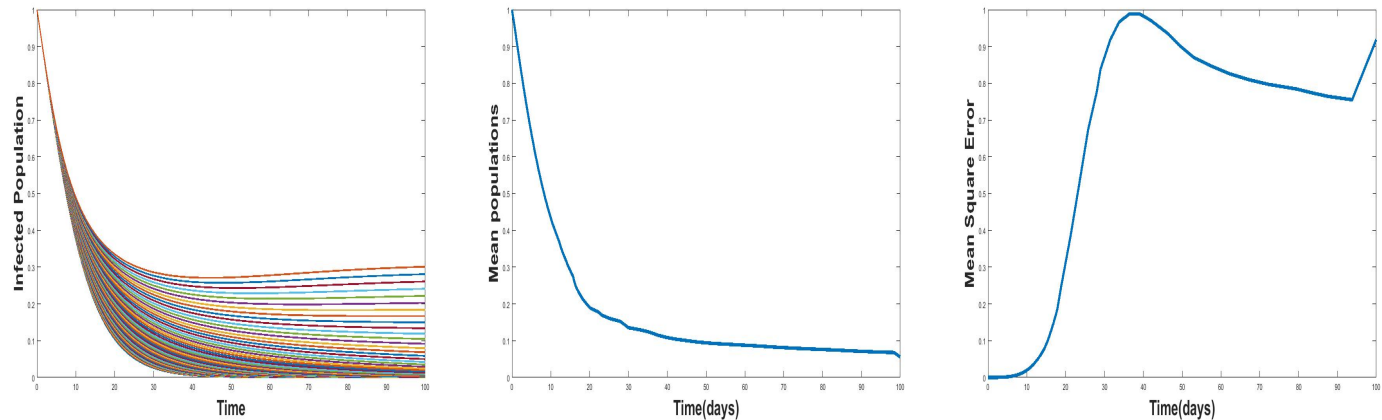

(a) Interval I : 0 to 2
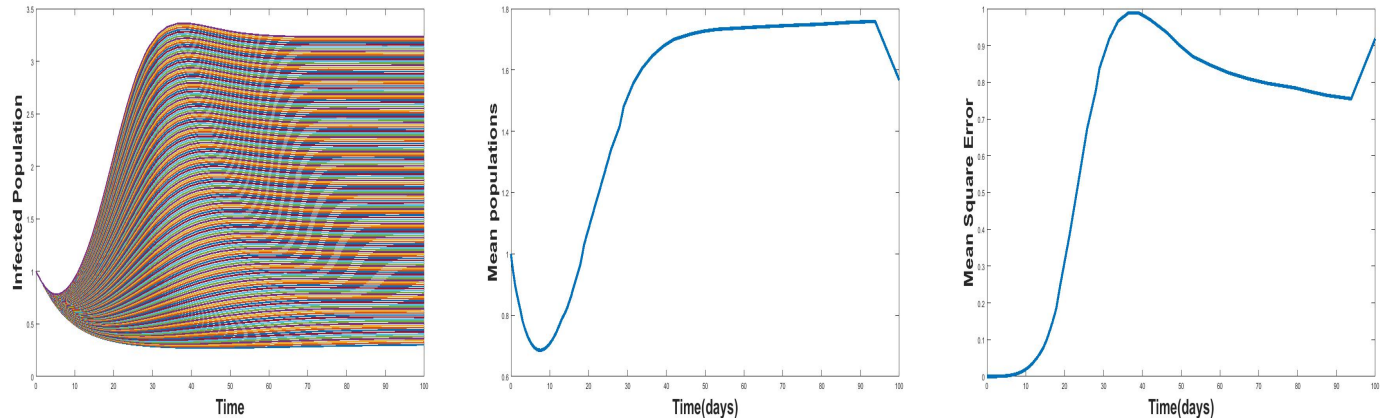

(b) Interval II : 2 to 4

Figure 5: Figure depicting the sensitivity Analysis of $\omega$ varied in two intervals in table 2. The plots depict the infected population for each varied value of the parameter $\omega$ per interval along with the mean infected population and the mean square error in the same interval.

\subsection{Parameter $\mu$}

The results related to sensitivity of $\mu$, varied in two intervals as mentioned in table 2, are given in Figure 6. The plots of infected population for each varied value of the parameter $\mu$ per interval, the mean infected population and the mean square error are used to determine the sensitivity. We conclude from these plots that the parameter $\mu$ is sensitive in interval I and insensitive in II. In similar lines, the sensitivity analysis is done for other parameters. 

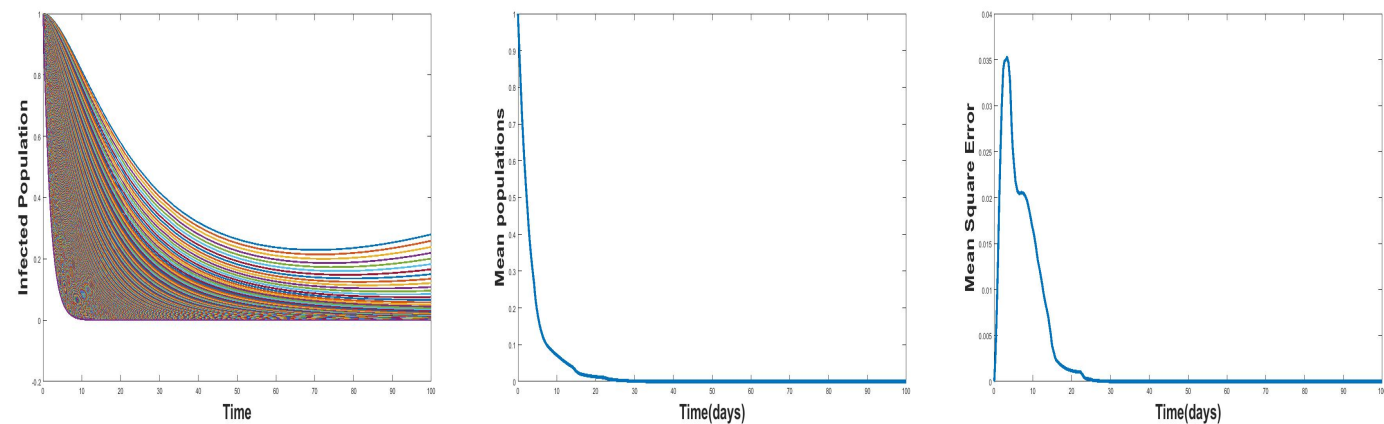

(a) Interval I: 0 to 0.5
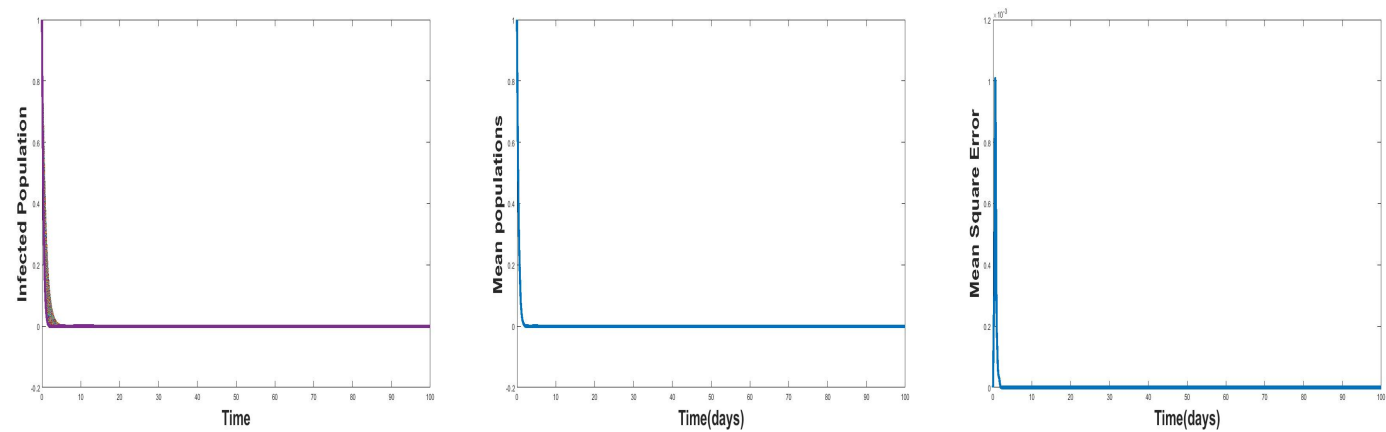

(b) Interval II : 0.5 to 2.5

Figure 6: Figure depicting the sensitivity Analysis of $\mu$ varied in two intervals in table 2 . The plots depict the infected population for each varied value of the parameter $\mu$ per interval along with the mean infected population and the mean square error in the same interval.

The summary of sensitivity analysis is given in Table 3. Due to brevity, the detailed sensitivity analysis of the rest of the parameters is given in Appendix 1.

In Figure 7 and Figure 8 the dynamics of the system for two specific values of $\beta$. i.e., 0.5 and 1 is plotted. 
Table 3: Summary of Sensitivity Analysis

\begin{tabular}{|l|l|l}
\hline Parameter & Interval & Step Size \\
\hline$\mu$ & 0 to 0.5 & $\checkmark$ \\
\cline { 2 - 3 } & .5 to 2.5 & $\times$ \\
\hline$\alpha$ & 0 to 0.5 & $\checkmark$ \\
\cline { 2 - 3 } & 2 to 3 & $\times$ \\
\hline$\gamma$ & 0 to 1 & $\times$ \\
\cline { 2 - 3 } & 2 to 5 & $\times$ \\
\hline$\epsilon$ & 0 to 1 & $\checkmark$ \\
\cline { 2 - 3 } & 1 to 2.5 & $\times$ \\
\hline$\omega$ & 0 to 0.5 & $\checkmark$ \\
\cline { 2 - 3 } & 1.5 to 2.5 & $\times$ \\
\hline$\delta$ & 0 to 2 & $\checkmark$ \\
\cline { 2 - 3 } & 2 to 4 & $\checkmark$ \\
\hline \multirow{5}{*}{$\delta$} & 0 to 1 & $\times$ \\
\cline { 2 - 3 } & 1 to 2.5 & $\times$ \\
\hline
\end{tabular}

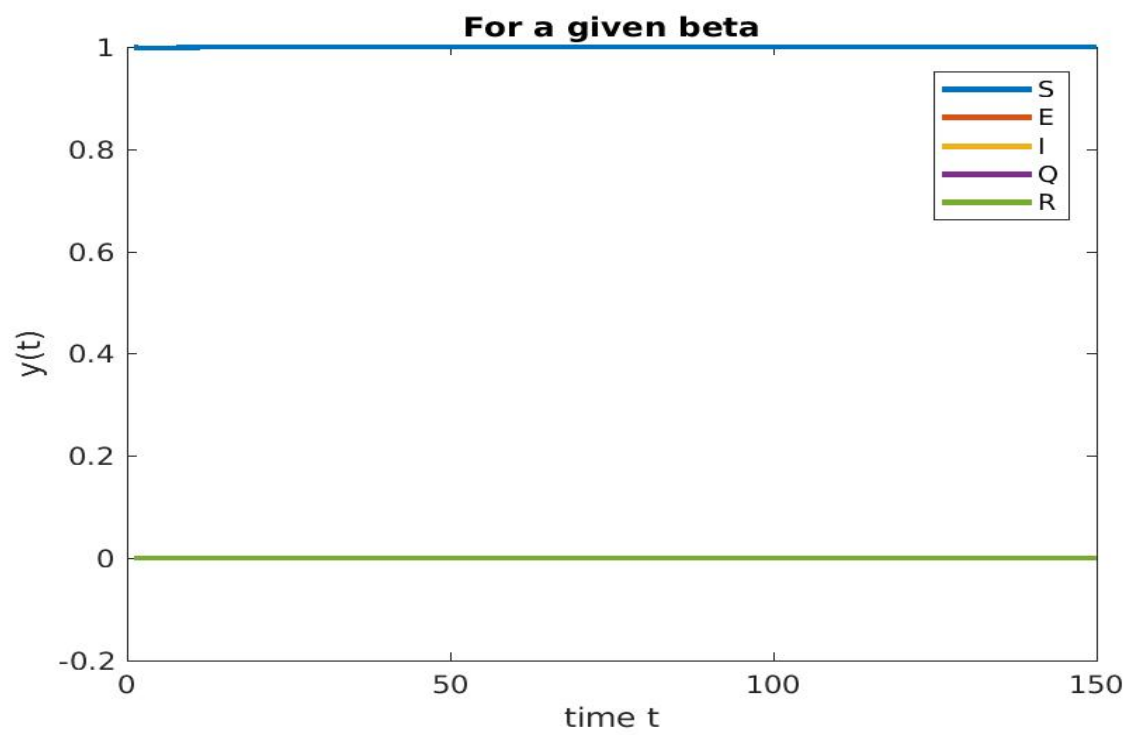

Figure 7: System dynamics when $\mathrm{R}_{0}<1(\beta=0.5)$

\section{Numerical Simulations}

In this section with the help of the numerical simulations we verify our theoretical results. The chosen parameter values for the model are given in the Table 1 . We have used the MATLAB software for the simulation.

For the parameter values from Table 1, we find that $f_{1}>0$ and $(\mu+\delta \alpha)^{2}>\rho^{2}(1-\alpha)^{2}$. The values of $d_{2}^{2}$ was found to be 0.0026 and $e_{2}^{2}$ was found to be 0.00045 . Therefore the condition $d_{2}^{2}>e_{2}^{2}$ of Theorem 4.1 is satisfied. Now from Theorem 4.1, $E_{0}$ should be asymptotically stable for all the possible values of delay. Here we take two values of delay and check for the stability of $E_{0}$. From Figure 9 , Figure 10, we see that the infection free equilibrium $E_{0}$ is locally asymptotically stable. 


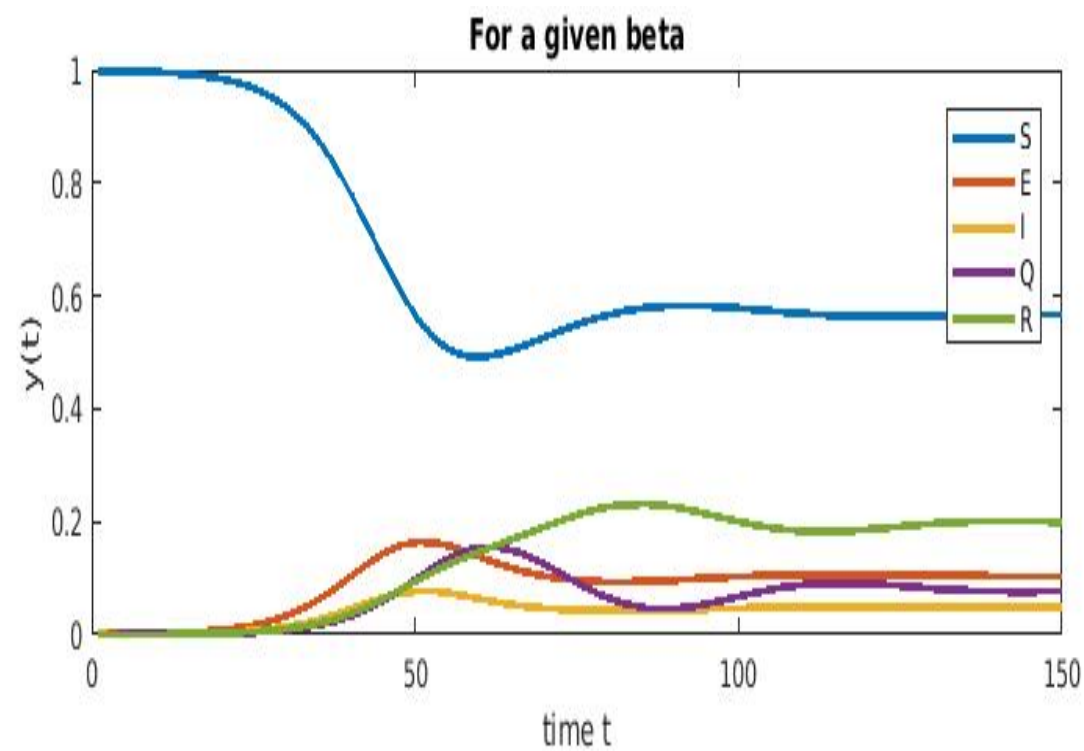

Figure 8: System dynamics when $\mathrm{R}_{0}>1(\beta=1.0)$

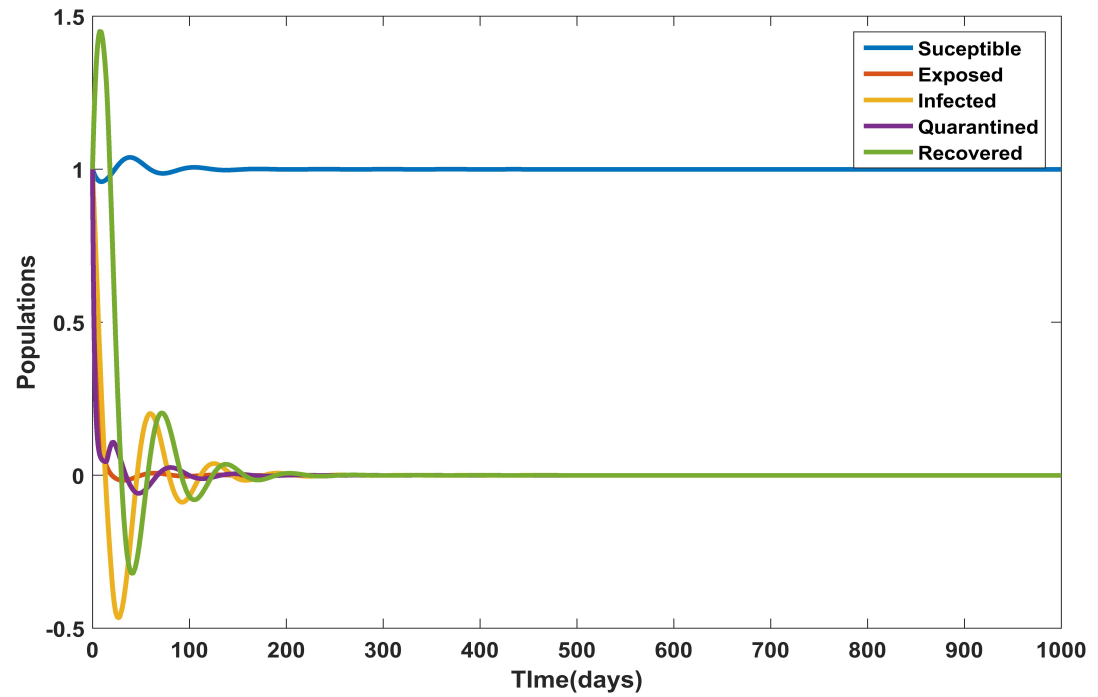

Figure 9: Stability of $E_{0}$ for $\tau^{\star}=5$

With the parameter values from Table 1, we have $d_{2}^{2}=0.0062$ and $e_{2}^{2}=0.0069$. Therefore we have $d_{2}^{2}<e_{2}^{2}$. Also $\gamma^{2}=0.0142>\omega^{2}=0.0139$ and $d_{2}=0.0128<\omega^{2}=0.0139$. We also see that the condition $x+y=9.4271>z=9.3946$ is satisfied. The critical value of delay $\tau^{\star}$ is calculated to be 5.8485 . Therefore from Theorem 4.1, we have $E_{0}$ to be asymptotically stable for $\tau<\tau^{\star}$ and unstable for $\tau>\tau^{\star}$. From Figure 11 , Figure 12, we see that $E_{0}$ is locally asymptotically stable for $\tau=5<\tau^{\star}=5.8485$ and unstable for $\tau=7>\tau^{\star}=5.8485$. We plot the bifurcation graph in Figure 13. From Figure 13, we see that the solutions of the system changes its nature from being asymptotically stable to unstable as the value of $\tau$ crosses 5.5. Therefore Hopf bifurcation occurs at $\tau^{\star}=5.8485$.

Now we numerically check the stability of infected equilibrium $E^{\star}$. For the parameter values from Table 1 , the infected equilibrium $E^{\star}$ was found to be $(0.633,0.093,0.08,0.003,0.16)$ and we calculate the values of $\beta \epsilon=.0882$ and $(\epsilon+\mu) \star\left(\gamma+\mu+\rho \exp ^{-\gamma \tau}\right)=.0559$. Therefore the existence of $E^{\star}$ is guaranteed. The values of $c_{1}, c_{2}, c_{3}$ were calculated to be $0.22,0.000035,0.0000025$ which are all positive. Also we calculate the 


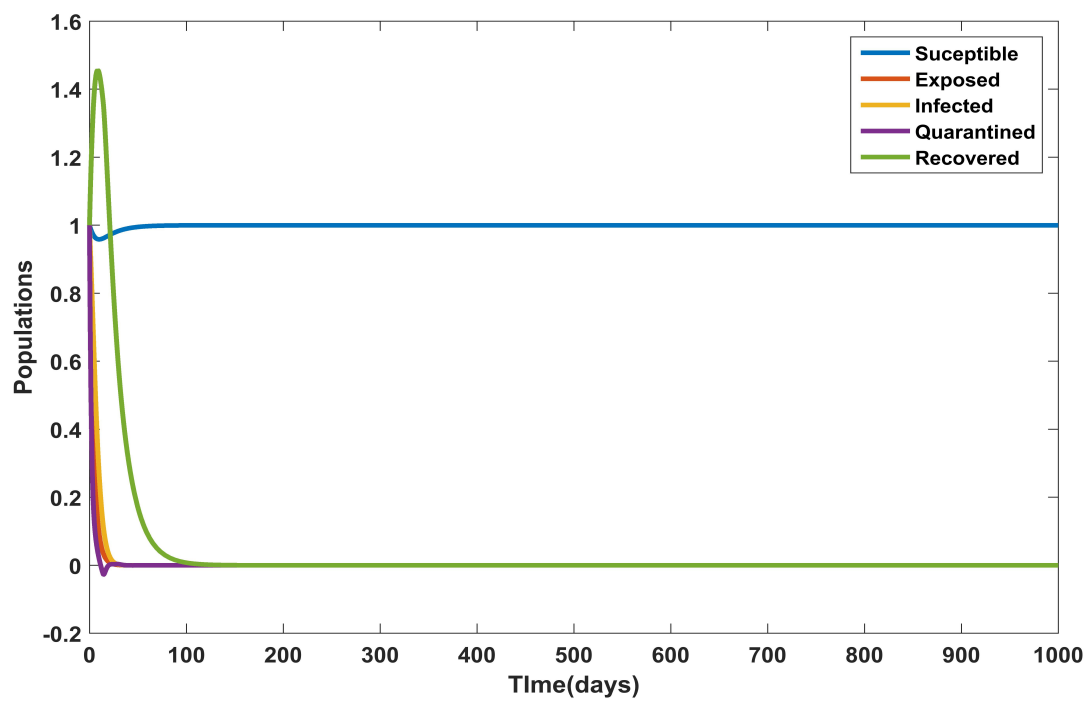

Figure 10: Stability of $E_{0}$ for $\tau^{\star}=20$

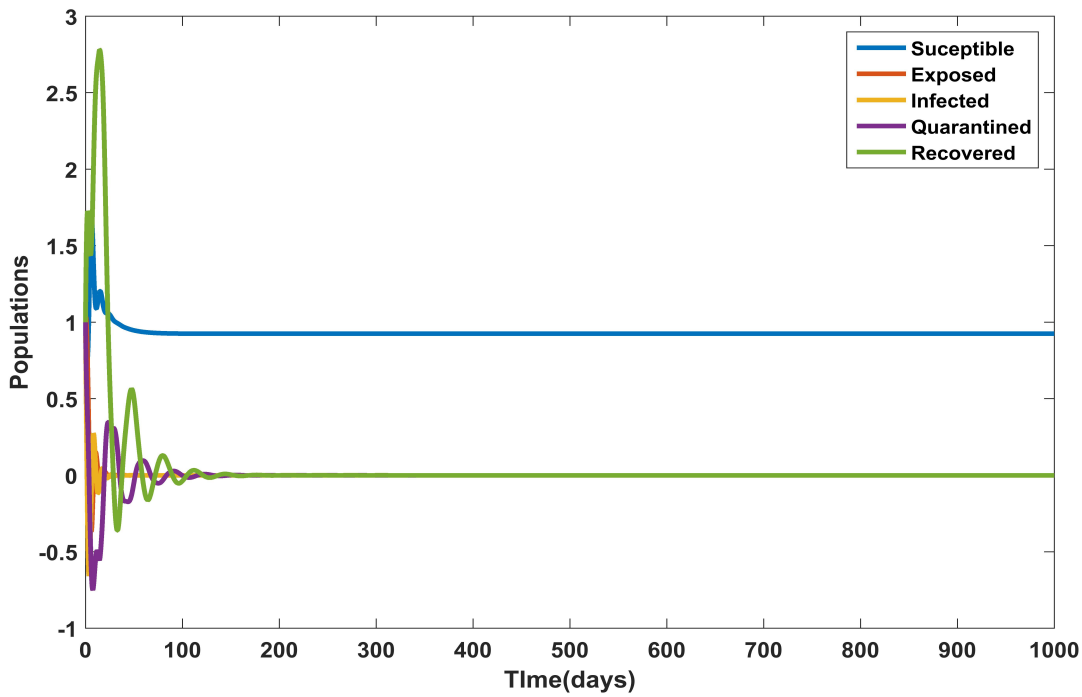

Figure 11: Stability of $E_{0}$ for $\tau=5<\tau^{\star}=5.8485$

values of $(\mu+\delta \alpha)^{2}$ to be 0.247 and value of $\rho^{2}(1-\alpha)^{2}$ to be 0.0017 and we see that $(\mu+\delta \alpha)^{2}>\rho^{2}(1-\alpha)$. Therefore from the stability analysis of $E^{\star}$ we must have $E^{\star}$ to be locally asymptotically stable for all delays. From Figure 14 and Figure 15, we see that $E^{\star}$ is stable for all delays.

When we take the value of $\alpha$ to be 0.001 we see that $(\mu+\delta \alpha)^{2}=0.004<\rho^{2}(1-\alpha)=0.005$. This violates the stability condition for $E^{\star}$ and we expect $E^{\star}$ to be unstable. From Figure 16, we see that $E^{\star}$ starts loosing its stability and starts becoming unstable when we have $(\mu+\delta \alpha)^{2}<\rho^{2}(1-\alpha)$. 


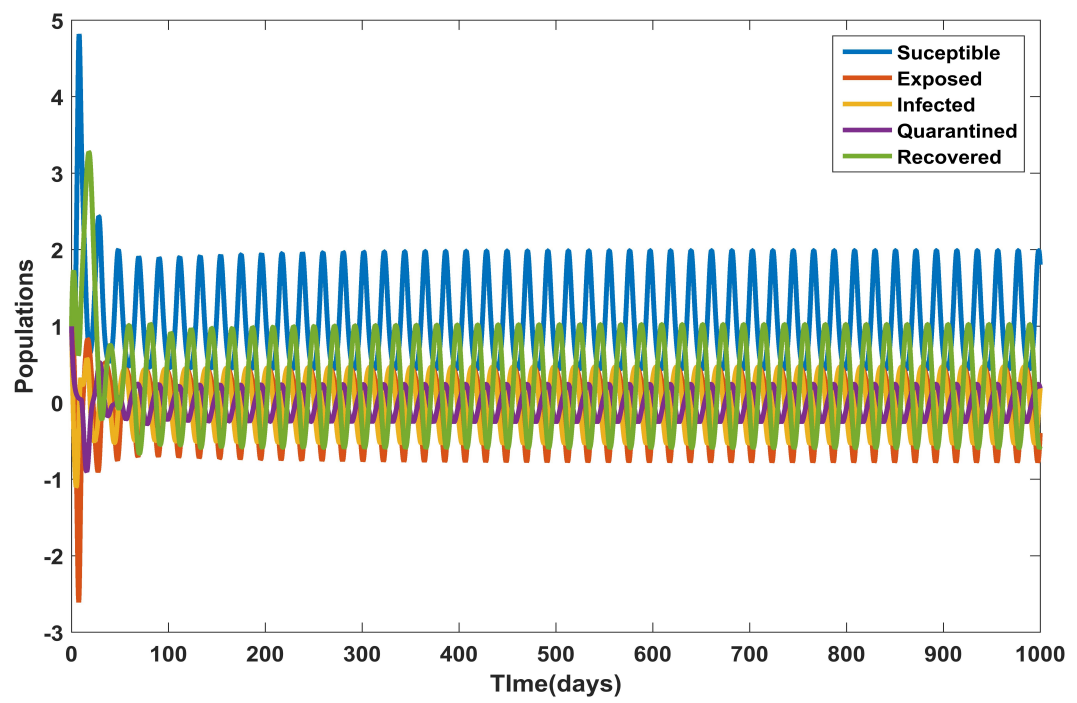

Figure 12: Stability of $E_{0}$ for $\tau=7>\tau^{\star}=5.8485$

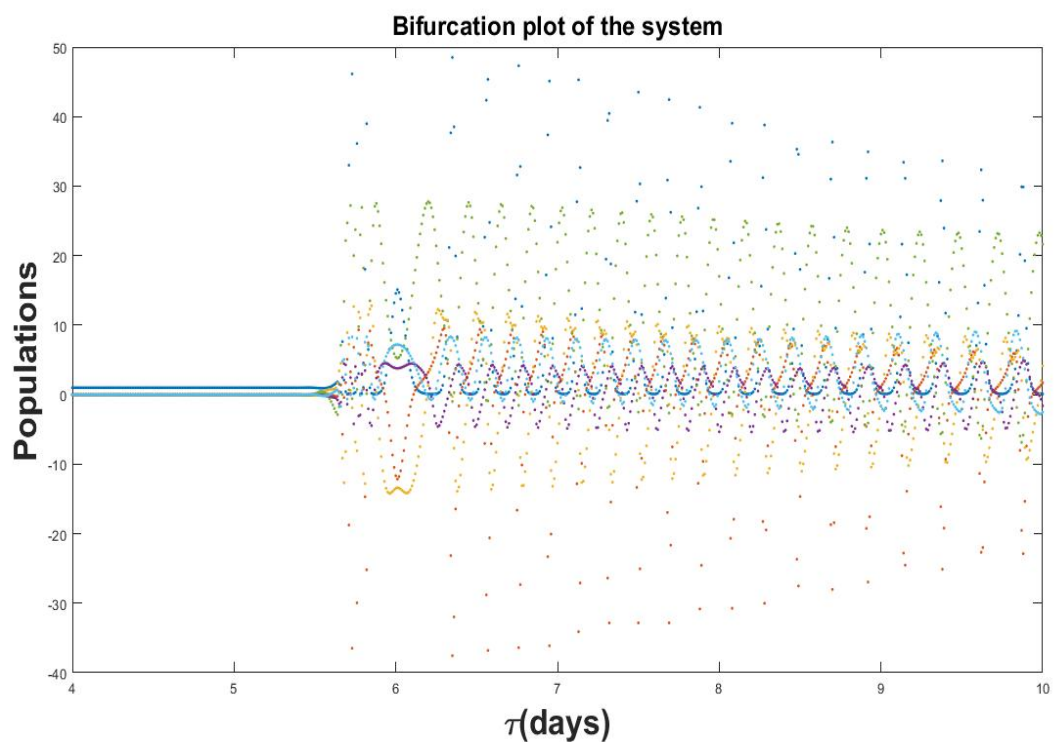

Figure 13: Stability of $E_{0}$ for $\tau^{\star}=5.8485$

\section{Effect of Temperature and Isolation}

\subsection{Impact of Temperature :}

Temperature is one of the key indicators that influence the spread of SARS-CoV-2 virus. For temperatures ranging from -10 to 50 , the average population belonging to each compartment is calculated using dde23 method in MATLAB. Since we represented the spread parameter $\beta$ as a linear and a quadratic function of temperature, the first subplot gives the average values with respect to $\beta$ as a linear function of temperature. And the second subplot gives the average values with respect to $\beta$ as a quadratic function of temperature. Both the subplots in Figures 17, 18, 19 concluded that with the decrease in temperature, the average number 


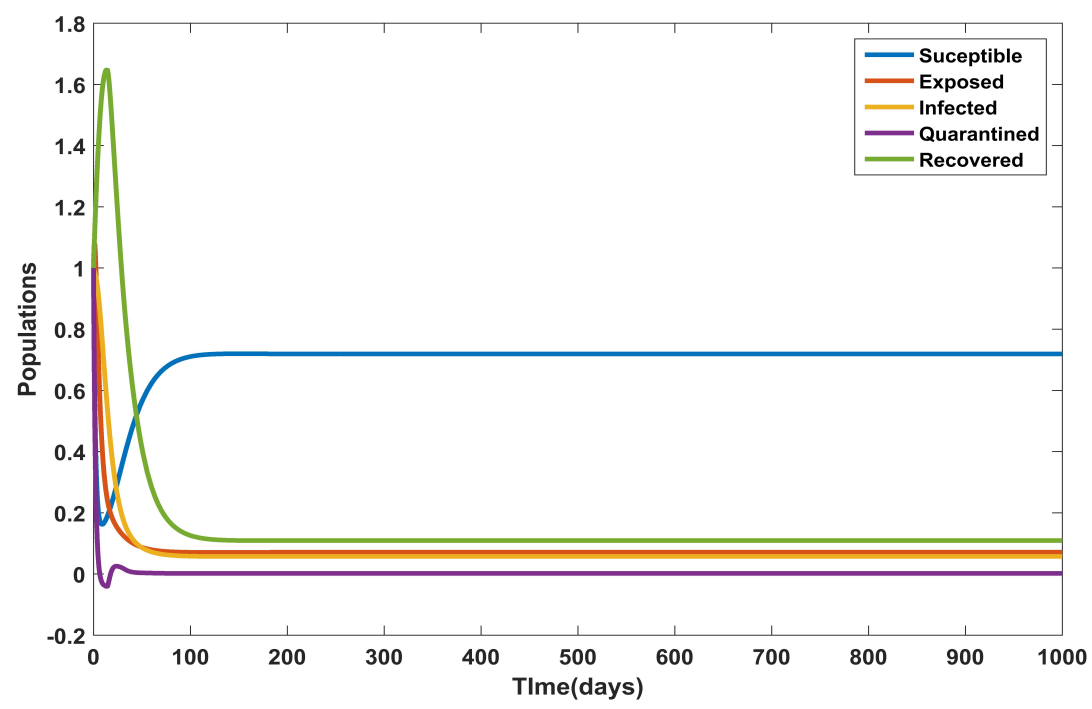

Figure 14: Stability of $E^{\star}$ independent of $\tau^{\star}$. Here we consider $\tau^{\star}=10$

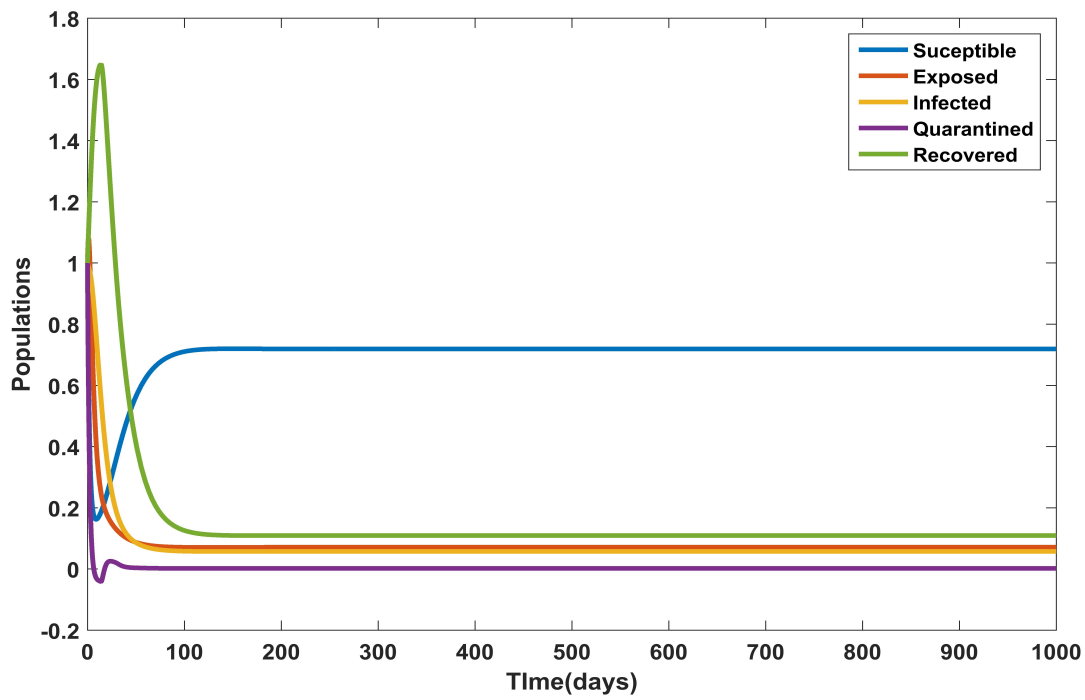

Figure 15: Stability of $E^{\star}$ independent of $\tau^{\star}$. Here we consider $\tau^{\star}=20$

of infections in a population of individuals increases. As the average temperature in India is in the interval $[-10,50]$ degrees Celsius, this work will be applicable to the entire country. 


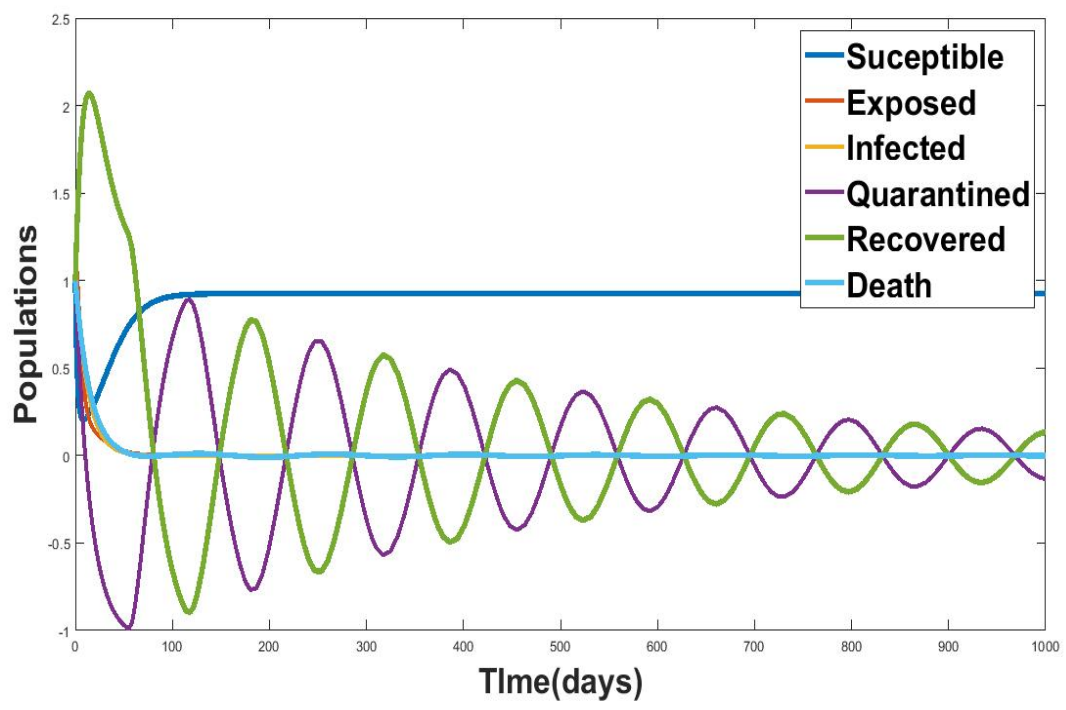

Figure 16: Stability of $E^{\star}$ for $\tau^{\star}=20$
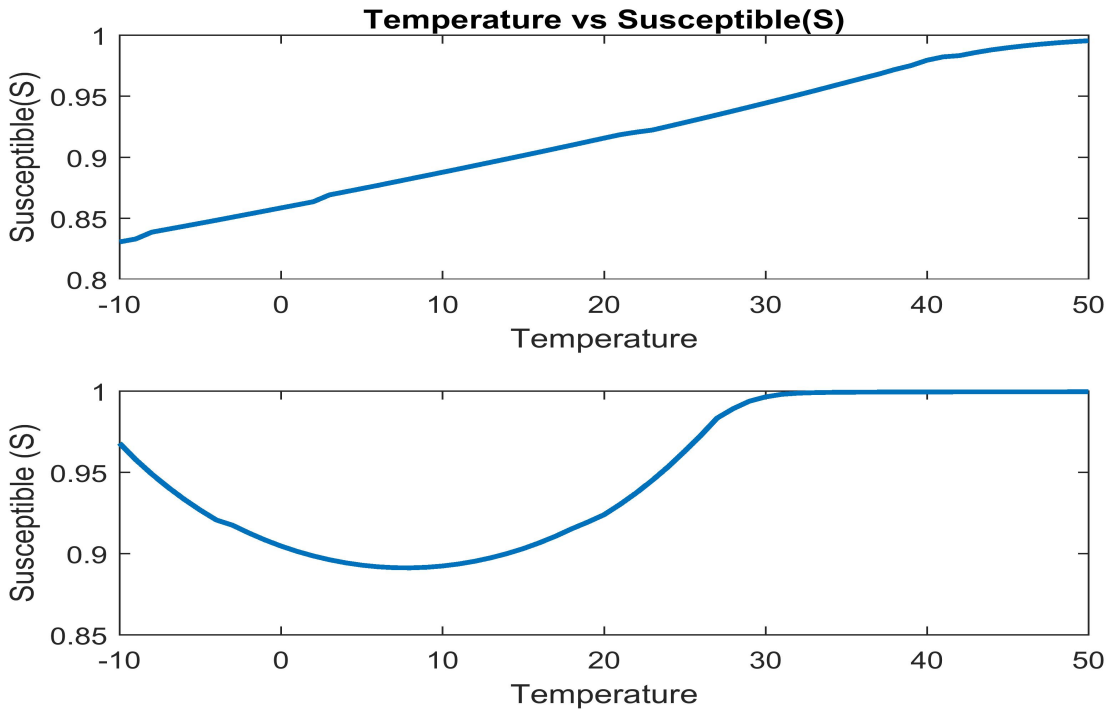

Figure 17: Susceptible vs temperature

\subsection{Impact of isolation :}

Isolation of infected individuals plays a very crucial role in controlling the pandemic situations. With the infections highly probable to increase in the coming winter season, efficient isolation strategies play an important role in controlling the spike of new infections. In addition to the probability of isolation, the delay in isolating infected individuals also play very crucial role in controlling infections. Figure 20 and Figure 21 give an idea on the impact of isolation probability and isolation delay with respect to the new infections. 

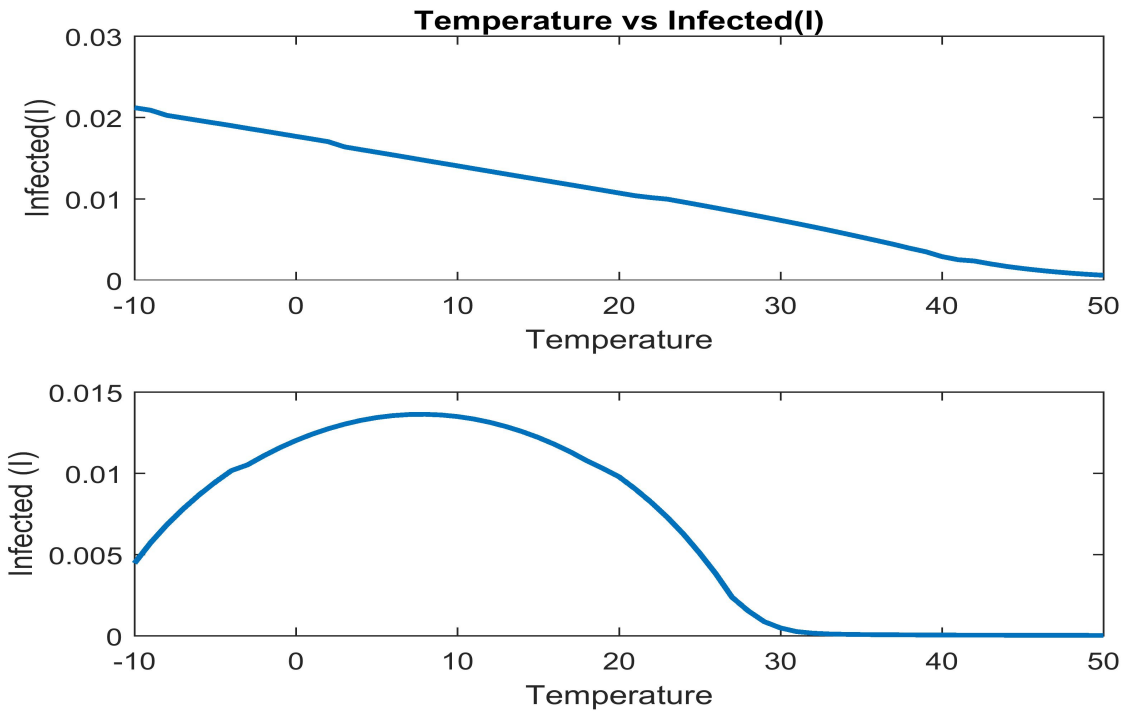

Figure 18: Infected vs temperature
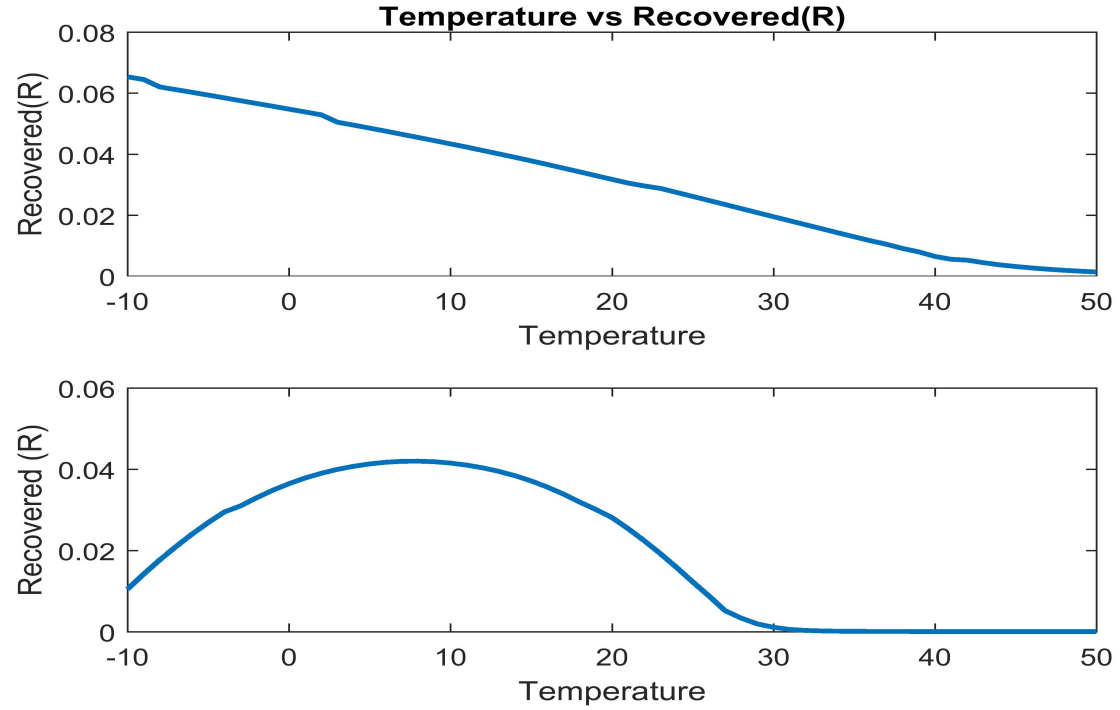

Figure 19: Recovered vs temperature

\section{Conclusions}

The system of delay differential equations proposed in this paper, incorporates key characteristics of COVID-19. This includes the impact of temperature on the spread parameter $\beta$ and the delay in isolating infected individuals. The reproduction number is calculated and the impact of temperature and two isolation indicators(isolation probability and isolation delay) with the temperature are plotted. The proposed model admits two equilibria, i.e., disease free equilibrium and infection equilibrium. The conditions for the stability of equilibrium points is obtained. The system exhibits hopf bifurcation. The critical delay at which hopf bifurcation occurs is calculated. Sensitivity analysis is performed for the proposed model. It is identified that a few parameters are sensitive only at some interval. The impact of temperature and isolation on COVID-19 is observed that with the decrease in temperature, the average number of infections and the average number of 


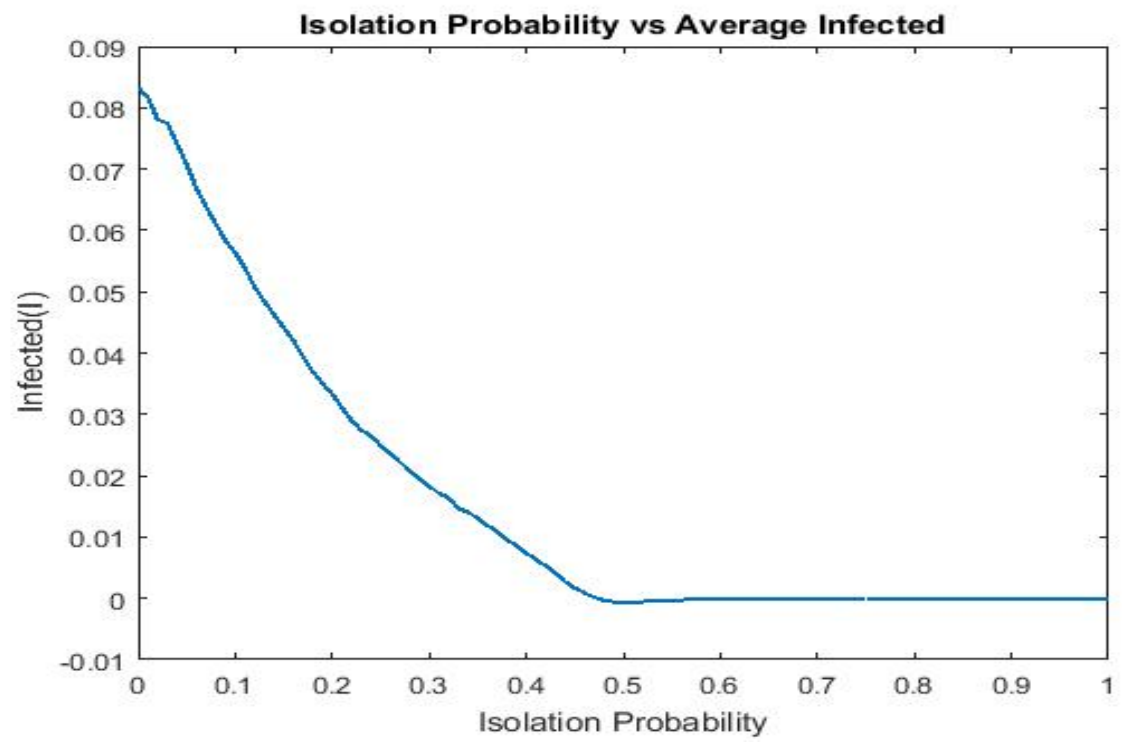

Figure 20: Isolation probability vs Average Infected

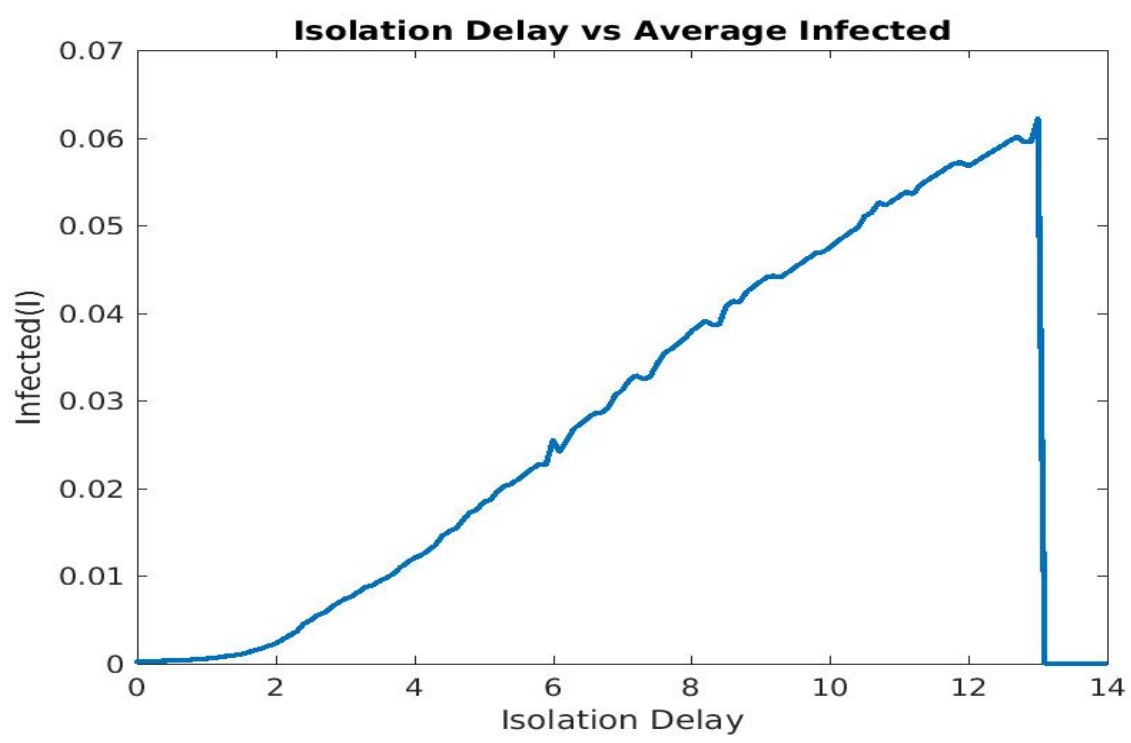

Figure 21: Isolation delay vs Average Infected

deaths increases. The conclusions obtained on the impact of temperature on COVID-19 is in similar lines with the observations in $[14,15,16]$. This observation is in similar lines with the conclusions in [17] that $60.0 \%$ of the confirmed cases of coronavirus disease 2019 (COVID-19) occurred in places where the air temperature ranged from $5 \mathrm{C}$ to $15 \mathrm{C}$, with a peak in cases at $11.54 \mathrm{C}$. With the winter season round the corner, these results warn us of an increase in daily new infected cases and possibly a second wave of COVID-19. Hence, the measures of face masks, sanitizers and social distancing has to be strictly followed in the coming future to avoid any sudden spike of cases.

Acknowledgements: The authors dedicate this paper to the founder chancellor of SSSIHL, Bhagawan Sri Sathya Sai Baba. The corresponding author also dedicates this paper to his loving elder brother D. A. C. Prakash who still lives in his heart. 
Financial Support: This research received no specific grant from any funding agency, commercial or nonprofit sectors.

Conflict of Interests Statement: The authors have no conflicts of interest to disclose.

Ethics Statement: This research did not require ethical approval.

\section{References}

[1] K. Sarkar, S. Khajanchi, J. J. Nieto, Modeling and forecasting the covid-19 pandemic in india, Chaos, Solitons \& Fractals 139 (2020) 110049.

[2] K. Chatterjee, K. Chatterjee, A. Kumar, S. Shankar, Healthcare impact of covid-19 epidemic in india: A stochastic mathematical model, Medical Journal Armed Forces India.

[3] A. Mahajan, N. A. Sivadas, R. Solanki, An epidemic model sipherd and its application for prediction of the spread of covid-19 infection in india, Chaos, Solitons \& Fractals 140 (2020) 110156.

[4] P. Samui, J. Mondal, S. Khajanchi, A mathematical model for covid-19 transmission dynamics with a case study of india, Chaos, Solitons \& Fractals 140 (2020) 110173.

[5] S. Saha, G. P. Samanta, J. J. Nieto, Epidemic model of covid-19 outbreak by inducing behavioural response in population, Nonlinear dynamics 102 (1) (2020) 455-487.

[6] S. Saha, G. P. Samanta, Modelling the role of optimal social distancing on disease prevalence of covid-19 epidemic, International journal of dynamics and control (2020) 1-25.

[7] M. Das, G. P. Samanta, Optimal control of fractional order covid-19 epidemic spreading in japan and india 2020, Biophysical Reviews and Letters 15 (04) (2020) 207-236.

[8] M. Das, G. P. Samanta, Stability analysis of a fractional ordered covid-19 model, Computational and Mathematical Biophysics 9 (1) (2021) 22-45.

[9] A. ul Rehman, R. Singh, P. Agarwal, Modeling, analysis and prediction of new variants of covid-19 and dengue co-infection on complex network, Chaos, Solitons \& Fractals (2021) 111008.

[10] N. Sharma, R. Singh, J. Singh, O. Castillo, Modeling assumptions, optimal control strategies and mitigation through vaccination to zika virus, Chaos, Solitons \& Fractals 150 (2021) 111137.

[11] P. Agarwal, R. Singh, A. ul Rehman, Numerical solution of hybrid mathematical model of dengue transmission with relapse and memory via ada-bashforth-moulton predictor-corrector scheme, Chaos, Solitons \& Fractals 143 (2021) 110564.

[12] P. Agarwal, R. Singh, Modelling of transmission dynamics of nipah virus (niv): a fractional order approach, Physica A: Statistical Mechanics and its Applications 547 (2020) 124243.

[13] N. Sharma, R. Singh, R. Pathak, Modeling of media impact with stability analysis and optimal solution of seirs epidemic model, Journal of Interdisciplinary Mathematics 22 (7) (2019) 1123-1156.

[14] K. Azuma, N. Kagi, H. Kim, M. Hayashi, Impact of climate and ambient air pollution on the epidemic growth during covid-19 outbreak in japan, Environmental research 190 (2020) 110042.

[15] M. F. Bashir, K. Shahzad, B. Komal, M. A. Bashir, M. Bashir, D. Tan, T. Fatima, U. Numan, et al., Environmental quality, climate indicators, and covid-19 pandemic: insights from top 10 most affected states of the usa, Environmental Science and Pollution Research (2021) 1-10.

[16] Á. Briz-Redón, Á. Serrano-Aroca, The effect of climate on the spread of the covid-19 pandemic: A review of findings, and statistical and modelling techniques, Progress in Physical Geography: Earth and Environment 44 (5) (2020) 591-604.

[17] Z. Huang, J. Huang, Q. Gu, P. Du, H. Liang, Q. Dong, Optimal temperature zone for the dispersal of covid-19, Science of The Total Environment (2020) 139487.

[18] J. Demongeot, Y. Flet-Berliac, H. Seligmann, Temperature decreases spread parameters of the new covid-19 case dynamics, Biology 9 (5) (2020) 94.

[19] A. Notari, Temperature dependence of covid-19 transmission, arXiv preprint arXiv:2003.12417.

[20] S. Kumar, Effect of meteorological parameters on spread of covid-19 in india and air quality during lockdown, Science of the Total Environment 745 (2020) 141021.

[21] L.-S. Young, S. Ruschel, S. Yanchuk, T. Pereira, Consequences of delays and imperfect implementation of isolation in epidemic control, Scientific reports 9 (1) (2019) 1-9.

[22] K. Maki, A delayed seiqr epidemic model of covid-19 in tokyo, medRxiv.

[23] P. Shi, Y. Dong, H. Yan, X. Li, C. Zhao, W. Liu, M. He, S. Tang, S. Xi, The impact of temperature and absolute humidity on the coronavirus disease 2019 (covid-19) outbreak-evidence from china, MedRxiv.

[24] J. A. Dieudonne, Foundations of Modern Analysis, Academic Press, New York, 1960. 


\section{Appendix 1: Sensitivity Analysis for other parameters}

$\alpha$
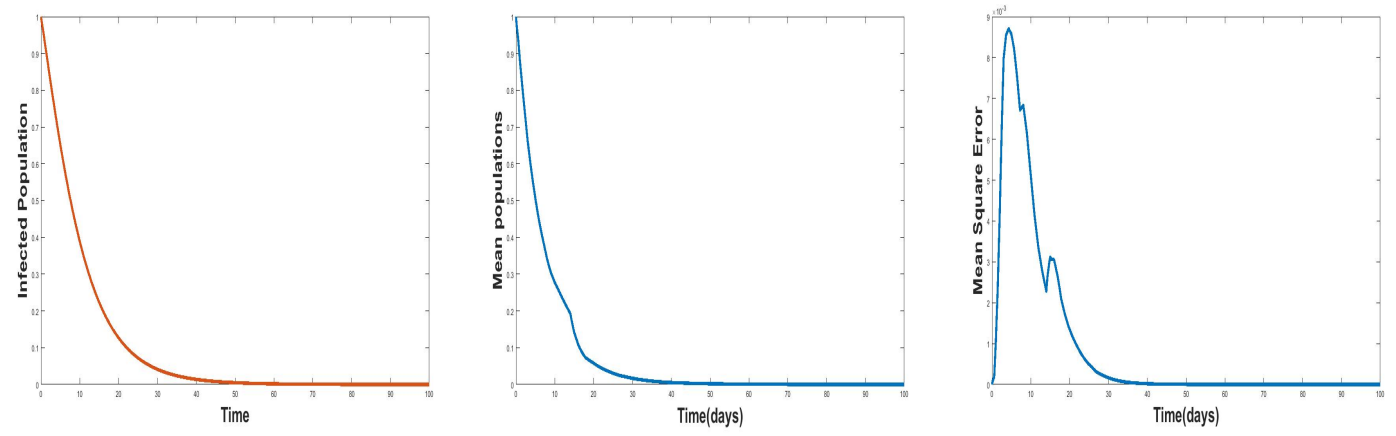

(a) Interval I: 0 to 3
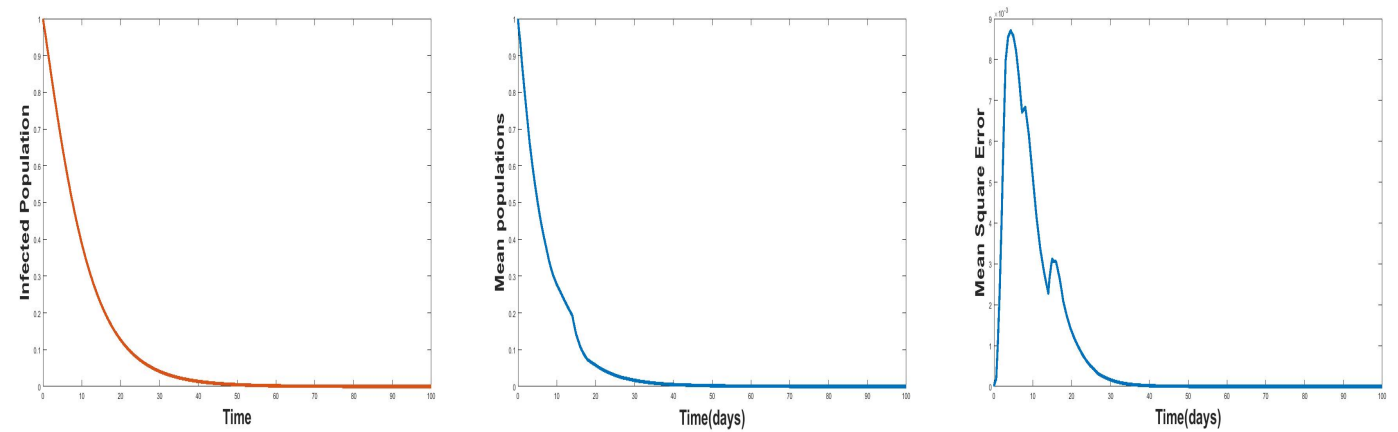

(b) Interval II : 3 to 5

Figure 22: Sensitivity Analysis of $\alpha$ 
172 D Bhanu Prakash et al.

Parameter $\boldsymbol{\epsilon}$

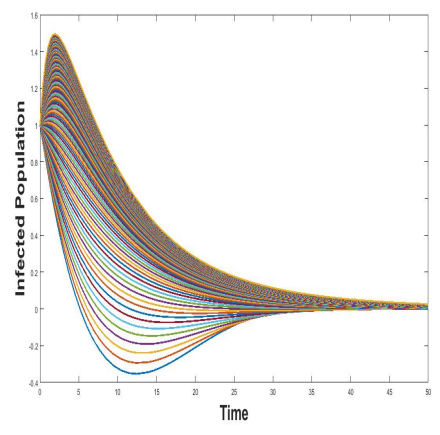

(a) Interval I : 0 to 0.5

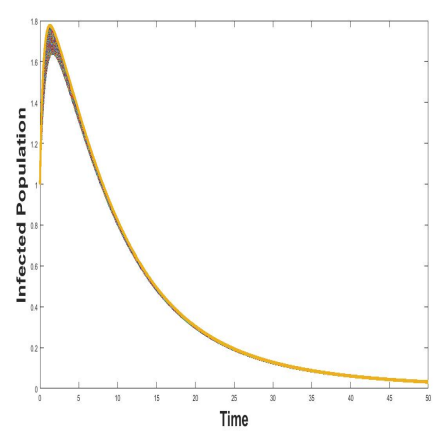

(b) Interval II : 1.5 to 2.5

Figure 23: Sensitivity Analysis of $\epsilon$
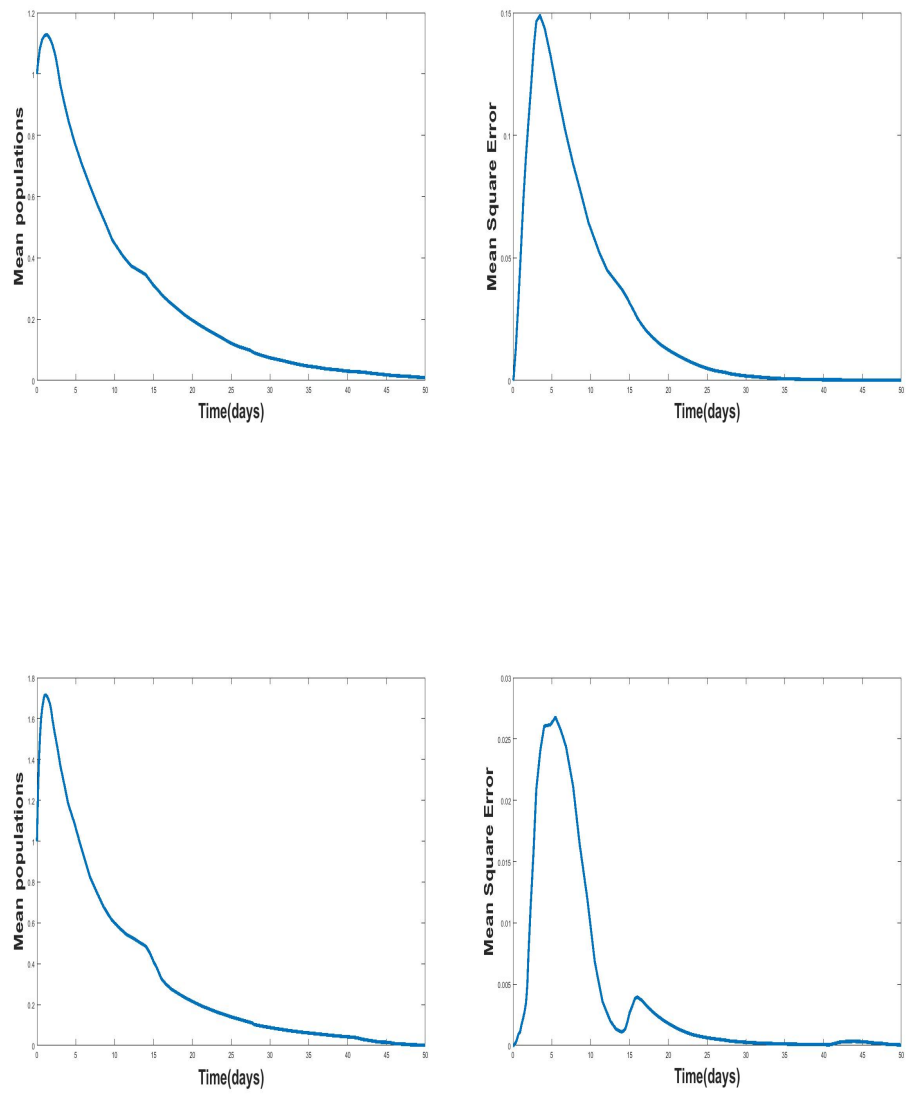
DE GRUYTER

Low temperatures or high isolation delay increases the average COVID-19 infections in India

173

Parameter $\boldsymbol{\delta}$
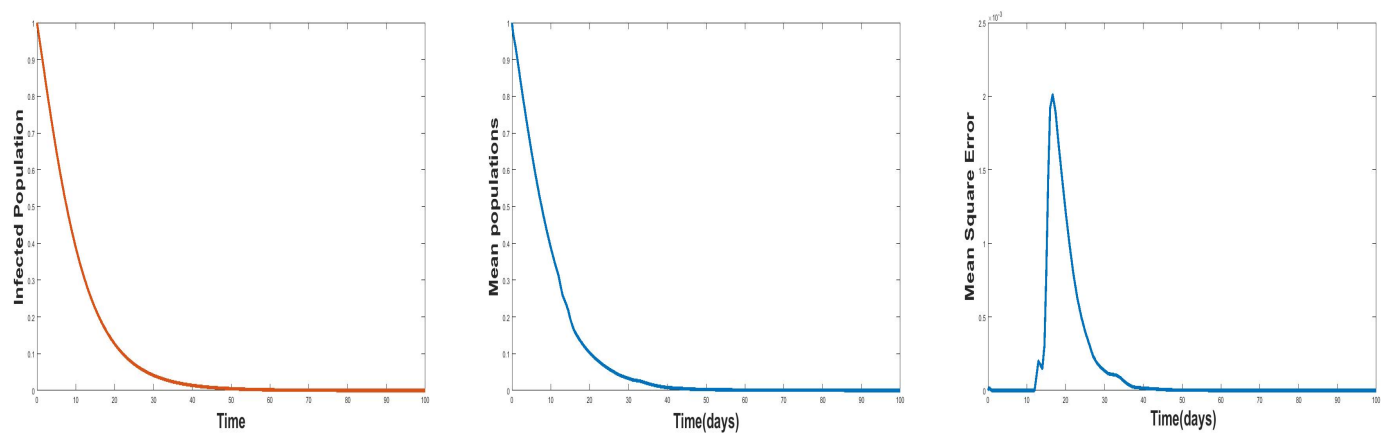

(a) Interval I : 0 to 1
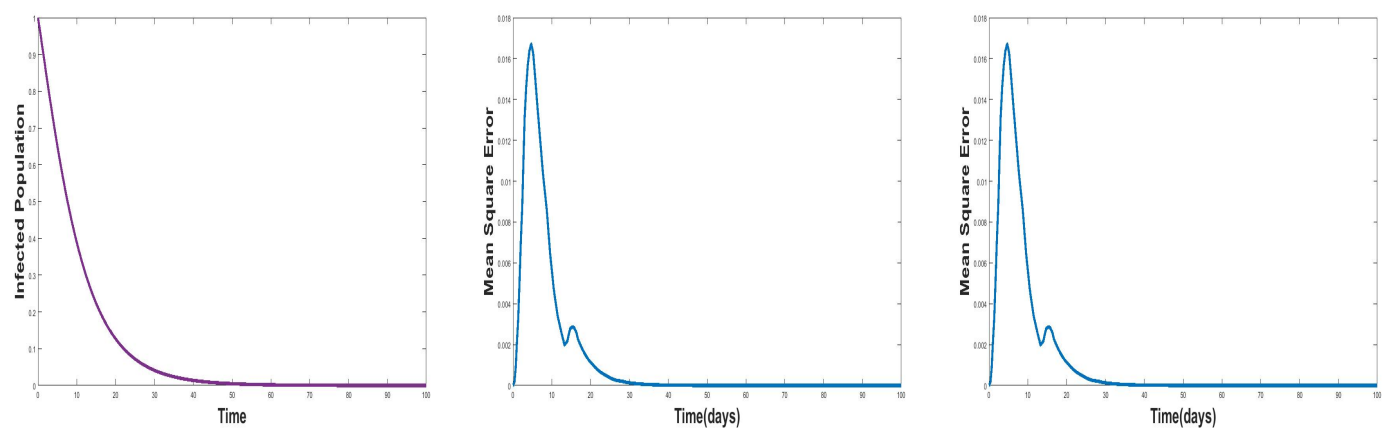

(b) Interval II : 1 to 2.5

Figure 24: Sensitivity Analysis of $\delta$ 


\section{Parameter $\gamma$}
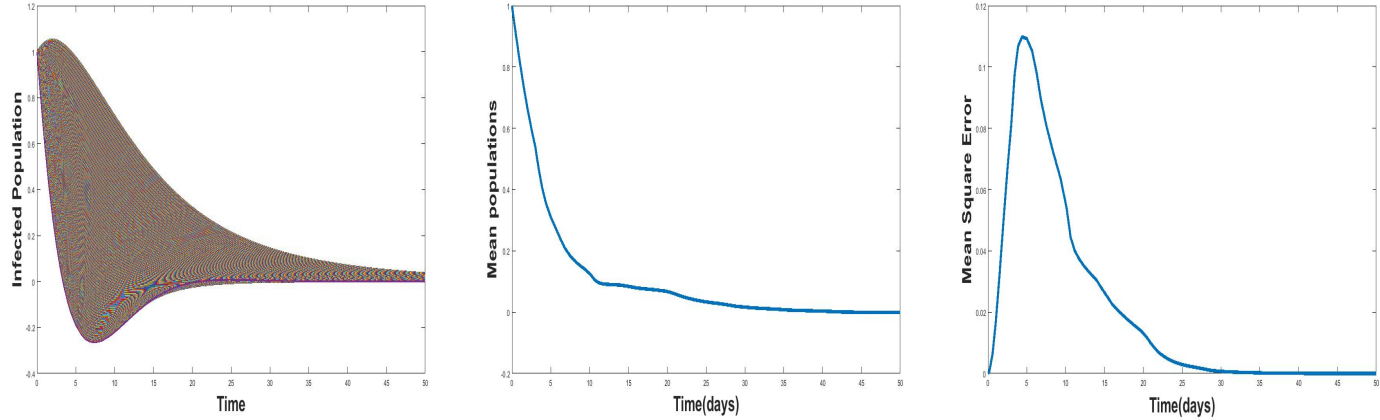

(a) Interval I : 0 to 1
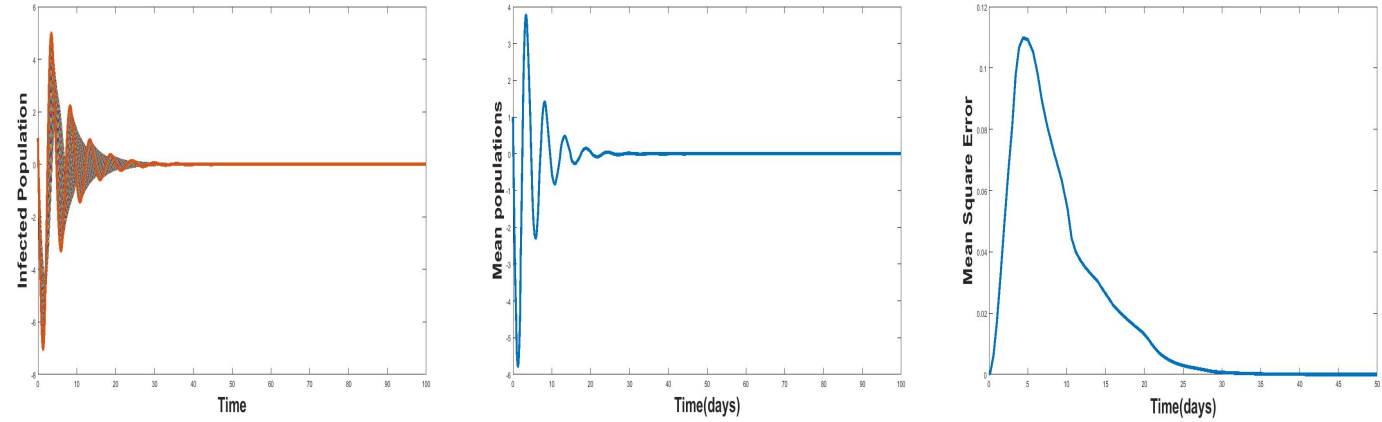

(a) Interval II : 1 to 2.5

Figure 25: Sensitivity Analysis of $\gamma$ 